\title{
On the initiation of surface waves by turbulent shear flow
}

Article

Accepted Version

Teixeira, M. A. C. and Belcher, S. E. (2006) On the initiation of surface waves by turbulent shear flow. Dynamics of Atmospheres and Oceans, 41 (1). pp. 1-27. ISSN 0377-0265 doi: https://doi.org/10.1016/j.dynatmoce.2005.10.001 Available at https://centaur.reading.ac.uk/5484/

It is advisable to refer to the publisher's version if you intend to cite from the work. See Guidance on citing.

To link to this article DOI: http://dx.doi.org/10.1016/j.dynatmoce.2005.10.001

Publisher: Elsevier

All outputs in CentAUR are protected by Intellectual Property Rights law, including copyright law. Copyright and IPR is retained by the creators or other copyright holders. Terms and conditions for use of this material are defined in the End User Agreement.

\section{www.reading.ac.uk/centaur}

\section{CentAUR}

Central Archive at the University of Reading

Reading's research outputs online 


\title{
On the initiation of surface waves by turbulent shear flow
}

\author{
M. A. C. Teixeira ${ }^{a}{ }^{*}$, S. E. Belcher ${ }^{b}$ \\ ${ }^{a}$ Centro de Geofísica da Universidade de Lisboa, Edifício C8, Campo Grande, \\ 1749-016 Lisbon, Portugal \\ ${ }^{\mathrm{b}}$ Department of Meteorology, University of Reading, Earley Gate, P.O. Box 243, \\ Reading RG6 6BB, U.K.
}

\begin{abstract}
An analytical model is developed for the initial stage of surface wave generation at an air-water interface by a turbulent shear flow in either the air or in the water. The model treats the problem of wave growth departing from a flat interface and is relevant for small waves whose forcing is dominated by turbulent pressure fluctuations. The wave growth is predicted using the linearised and inviscid equations of motion, essentially following Phillips (1957), but the pressure fluctuations that generate the waves are treated as unsteady and related to the turbulent velocity field using the rapid-distortion treatment of Durbin (1978). This model, which assumes a constant mean shear rate $\Gamma$, can be viewed as the simplest representation of an oceanic or atmospheric boundary layer.

For turbulent flows in the air and in the water producing pressure fluctuations of similar magnitude, the waves generated by turbulence in the water are found to be considerably steeper than those generated by turbulence in the air. For resonant waves, this is shown to be due to the shorter decorrelation time of turbulent pressure in the air (estimated as $\propto 1 / \Gamma$ ), because of the higher shear rate existing in the air flow, and due to the smaller length scale of the turbulence in the water. Nonresonant waves generated by turbulence in the water, although being somewhat
\end{abstract}


gentler, are still steeper than resonant waves generated by turbulence in the air. Hence, it is suggested that turbulence in the water may have a more important role than previously thought in the initiation of the surface waves that are subsequently amplified by feedback instability mechanisms.

Key words: Surface water waves; Turbulent shear flow; Wave generation; Resonance; Rapid distortion theory

\section{Introduction}

There are many types of interaction between turbulent flows and free surfaces, or at interfaces between two fluids of very different densities. It is useful to consider these interactions in three categories:

(1) Turbulent Reynolds stresses act on the mean flow and thence change the interactions of the mean flow with the interface. The growth of surface waves by wind forcing, for example, is usually understood in these terms (e.g. Belcher and Hunt 1998).

(2) The presence of the interface and waves on the interface change the turbulence. For example, when the Froude number is large, the free surface inhibits vertical motion in the turbulence over a depth comparable with the integral scale of the turbulence, and redistributes the energy into horizontal fluctuations (Hunt and Graham 1978; Magnaudet 2003; Pan and Bannerjee 1995). When the free surface also carries a progressive surface wave there is additional distortion of the turbulence by the periodic straining motion of the wave and, over longer times, by the rectified straining by the Stokes drift associated with the wave (Teixeira and

\footnotetext{
* Corresponding author.

Email addresses: mateixeira@fc.ul.pt (M. A. C. Teixeira),

s.e.belcher@reading.ac.uk (, S. E. Belcher).
} 
Belcher 2002). This long time straining leads to elongated streamwise vortices, reminiscent of Langmuir circulations observed in the oceans, as reviewed recently by Thorpe (2004). When the waves become very steep they may break and generate turbulence themselves, as shown by the detailed measurements of Gemmrich and Farmer (2004).

(3) Thirdly, when the turbulence is more energetic, so that the Froude number is nearer one, there is the possibility that it will generate waves on the interface. Very energetic motions churn and possibly break up the interface into drops (Brocchini and Peregrine 2001). But weaker turbulent motions can also yield order one deformations. These deformations may correspond to forced or free waves. Subsurface turbulence manifests itself through forced (i.e. non-resonant) waves whose elevation can be estimated from the hydrostatic relation as $\eta \approx p /\left(\rho_{w} g\right)$, where $p$ is the magnitude of the associated pressure fluctuations, $\rho_{w}$ is the density of water and $g$ is the acceleration of gravity. But Phillips (1957) showed that when pressure fluctuations associated with the turbulence advect along the interface at the phase speed of free surface waves there is a resonant forcing of free surface modes, which then grow indefinitely.

In this paper we consider further the third category, namely waves generated on the interface, and we focus on the case of weak turbulent fluctuations. This parameter regime is of relevance to the ocean, where the Froude number of the turbulence is usually low.

Phillips (1957) derived an expression for the wave spectrum resulting from the resonance process mentioned above as a function of the wavenumber spectrum of the turbulent pressure fluctuations, and of their integral time scale. Hasselmann (1968) showed how the wave spectrum can be expressed as a function of the wavenumber-frequency spectrum of the turbulent pressure fluctuations evaluated at the resonance frequency. At the time of these pioneering investigations, little was known about turbulent pressure fluctuations. Later, 
Kitaigorodskii and Lumley (1983) and Sazontov and Shagalov (1984) used dimensional analysis to find consistent forms for the spectrum of the turbulent pressure fluctuations, and assumed simplified models of their spatio-temporal structure. This, with Phillips' theory, allowed calculation of wave spectra, but these turbulent pressure spectra contained arbitrary constants and were dependent on ambiguous scalings. In this sense, Phillips' theory can be considered incomplete.

Meanwhile, laboratory experiments of Gelci et al. (1985) and Giovanangeli and Memponteil (1985), which generated vortices in air flow above a water surface, have demonstrated action of the mechanism qualitatively. Kahma and Donelan (1988) used a homogeneous boundary layer flow similar to that assumed in Phillips' (1957) study. They measured the pressure frequency spectrum, but were also forced to make strong assumptions about the spatiotemporal structure, and consequently were cautious in commenting on the order-of-magnitude agreement achieved between the theory and their data.

The main focus of the theoretical treatment given here is therefore the turbulent pressure fluctuations that drive the surface waves. Now, in practice, the turbulence near the interface is usually maintained by a sheared mean flow. The turbulence is then distorted by the mean shear, and eddies that give rise to the surface pressure fluctuations undergo a lifecycle. The resonance condition that the pressure fluctuation has a length scale and advection speed that matches those of the free surface mode, is then satisfied for only a finite time, or is not even satisfied, if the flow speed is sufficiently low.

We illustrate this mechanism quantitatively here by considering turbulence in a mean flow with constant shear. The evolution of the turbulence is calculated using a linearised rapid distortion framework (hereafter RDT) that captures the early stages of the lifecycle of the turbulent eddies. RDT considers the distortion of the turbulence by the mean flow, whilst neglecting the nonlin- 
ear interactions of the turbulence with itself (Batchelor and Proudman 1954). In the case of shear flows, this condition is satisfied when the shear rate is large compared with the typical frequency of the turbulent eddies, a situation that occurs in turbulent boundary layers (Lee et al. 1990). Townsend (1970) showed that RDT captures with good accuracy the spatial correlation of the turbulent velocity in turbulent free shear layers. Subsequently, Lee and Hunt (1989) and Mann (1994) have shown how RDT is useful for evaluating velocity fluctuations in shear flows near a flat wall; Durbin (1978) calculated turbulent pressure fluctuations in a constant shear flow near a wall, that show encouraging agreement with measurements. Although a constant shear is a crude approximation to the mean velocity profile in the boundary layer, this good agreement may be due to the fact that the main contributions to the pressure at the wall come from a relatively thin region near the wall (sometimes called the buffer layer, see Chang et al. 1999).

Phillips' (1957) theory considers the problem of wave initiation, and the relevant pressure fluctuations in his analysis are those at a flat air-water interface. The pressure fluctuations at a shear-driven flat air-water interface are essentially similar to those at a flat wall, since these two boundaries only differ in the viscous coupling, which affects the pressure little. Here, then, Durbin's model of turbulence distorted by a constant shear near a rigid wall is adopted to calculate the evolution of the pressure fluctuations, which are then used to calculate surface wave generation in the framework of the theory developed by Phillips (1957), but focusing also on non-resonant waves. The model is used to illustrate: (i) how evolution of the pressure fluctuations limits their resonant generation of surface waves, (ii) the role of non-resonant pressure fluctuations in generating waves, and (iii) the relative effectiveness in generating waves of turbulent fluctuations in the air flow above, and in the water flow below, at an air-water interface. 
The remainder of this paper is organised as follows. In Section 2, the theoretical model for the initiation of surface waves by a turbulent shear flow will be presented. In Section 3, some statistics of the generated waves will be shown and their behaviour will be discussed in detail. Finally, in Section 4, the main conclusions of this paper will be reported.

\section{Theoretical model}

Consider two fluids, referred to as air and water, of densities $\rho_{a}$ and $\rho_{w}$, with $\rho_{a} / \rho_{w} \ll 1$. Initially their interface is at $x_{3}=0$. At $t=0$, a flow in either the water or the air, is suddenly started with mean component, $\vec{U}$, with constant shear maintained by some external force parallel to the interface. At $t=0$ there is also a turbulent component to the flow, $\vec{u}$, in either the air or the water with the mean shear (see Fig. 1). Symbolically, in a frame of reference moving with the mean velocity at the air-water interface,

$$
\vec{U}=\left(\Gamma x_{3}, 0,0\right), \quad \vec{u}=\left(u_{1}, u_{2}, u_{3}\right)
$$

where $\Gamma$ is the mean shear rate. The aim is then to calculate the evolution of the turbulent component of the flow. This evolution generates waves on the air-water interface, with an associated flow field,

$$
\vec{u}^{(W)}=\left(u_{1}^{(W)}, u_{2}^{(W)}, u_{3}^{(W)}\right)
$$

Consider evolution at very high Reynolds number, so that viscous stresses can be entirely neglected. Coupling at the air-water interface is then entirely through the pressure.

Here the evolution of $\vec{u}$ and $\vec{u}^{(W)}$ is calculated in the rapid distortion approximation (RDT), so that the equations of motion are linearised with respect to $\vec{u}$ and $\vec{u}^{(W)}$, which are assumed to be formally of the same order of magnitude. 
If the velocity and integral length scales of the initial turbulence are $u$ and $l$ respectively, then this RDT approximation is valid when

$$
\frac{\Gamma l}{u} \gg 1, \quad \Gamma t \leq 10
$$

The first condition states that straining of the turbulence by the mean shear is stronger than the straining of the turbulence by itself (Townsend 1976). As the distortion acts over time, the turbulence becomes increasingly anisotropic, and the linearisation ceases to be valid. The second condition is a limit on this anisotropy (Townsend 1976).

The equations governing momentum in the turbulent and wave fields are then given by

$$
\begin{aligned}
& \frac{\partial u_{i}}{\partial t}+U_{j} \frac{\partial u_{i}}{\partial x_{j}}+u_{j} \frac{\partial U_{i}}{\partial x_{j}}=-\frac{1}{\rho} \frac{\partial p}{\partial x_{i}}, \\
& \frac{\partial u_{i}^{(W)}}{\partial t}+U_{j} \frac{\partial u_{i}^{(W)}}{\partial x_{j}}+u_{j}^{(W)} \frac{\partial U_{i}}{\partial x_{j}}=-\frac{1}{\rho} \frac{\partial p^{(W)}}{\partial x_{i}} .
\end{aligned}
$$

The turbulence couples to the wave field through the boundary conditions.

The kinematic boundary condition at the air-water interface requires that the vertical velocity of the interface equals that of the adjacent fluid. The linearised kinematic boundary condition is

$$
u_{3}+u_{3}^{(W)}=\frac{\partial \zeta}{\partial t} \quad \text { on } x_{3}=0
$$

where $\zeta$ is the surface elevation. Since this equation is linear it can be satisfied separately by the turbulence and wave fields

$$
u_{3}=0, \quad u_{3}^{(W)}=\frac{\partial \zeta}{\partial t} \quad \text { on } x_{3}=0 .
$$

The first relation on the turbulence is just the blocking condition for turbulence near a flat boundary (Hunt and Graham 1978; Teixeira and Belcher 
2000). The second relation is the kinematic boundary condition for infinitesimal waves in the absence of turbulence. The initial condition is that there is a specified turbulent velocity field in either the air or the water. The interface and the wave motions are taken to be zero at $t=0$.

In this way we understand that the wave motion is associated with deformation of the free surface, and the turbulence with the evolution of the initial random velocity field. Coupling between these two random velocity fields arises through the final boundary condition, namely the dynamical boundary condition at the interface. This dynamical boundary condition is that the discontinuity of the pressure across the interface is due only to surface tension. It is formulated below.

\subsection{Fourier amplitudes}

The turbulent flow field that is imposed at $t=0$ is assumed to be homogeneous and isotropic. The boundary conditions are homogeneous in horizontal planes, and so is the mean flow. Although, as shown below, the boundary then makes the turbulence anisotropic in the vertical direction, the statistics of the flow remain homogeneous in the horizontal. Hence the turbulent velocity and pressure can be expressed as Fourier integrals along the horizontal directions, namely

$$
\begin{aligned}
& u_{i}(\vec{x}, t)=\iint \hat{u}_{i}\left(k_{1}, k_{2}, x_{3}, t\right) \mathrm{e}^{\mathrm{i}\left(k_{1} x_{1}+k_{2} x_{2}\right)} \mathrm{d} k_{1} \mathrm{~d} k_{2}, \\
& p(\vec{x}, t)=\iint \hat{p}\left(k_{1}, k_{2}, x_{3}, t\right) \mathrm{e}^{\mathrm{i}\left(k_{1} x_{1}+k_{2} x_{2}\right)} \mathrm{d} k_{1} \mathrm{~d} k_{2},
\end{aligned}
$$

where $\hat{u}_{i}$ and $\hat{p}$ are time dependent Fourier amplitudes and $\left(k_{1}, k_{2}\right)$ is the horizontal wavenumber vector. The surface elevation associated with the wave field generated by the turbulence, and the corresponding orbital velocity and pressure can be expressed similarly, 


$$
\begin{aligned}
\zeta\left(x_{1}, x_{2}, t\right) & =\iint \hat{\zeta}\left(k_{1}, k_{2}, t\right) \mathrm{e}^{\mathrm{i}\left(k_{1} x_{2}+k_{2} x_{2}\right)} \mathrm{d} k_{1} \mathrm{~d} k_{2}, \\
u_{i}^{(W)}(\vec{x}, t) & =\iint \hat{u}_{i}^{(W)}\left(k_{1}, k_{2}, x_{3}, t\right) \mathrm{e}^{\mathrm{i}\left(k_{1} x_{1}+k_{2} x_{2}\right)} \mathrm{d} k_{1} \mathrm{~d} k_{2}, \\
p^{(W)}(\vec{x}, t) & =\iint \hat{p}^{(W)}\left(k_{1}, k_{2}, x_{3}, t\right) \mathrm{e}^{\mathrm{i}\left(k_{1} x_{1}+k_{2} x_{2}\right)} \mathrm{d} k_{1} \mathrm{~d} k_{2},
\end{aligned}
$$

where $\hat{\zeta}, \hat{u}_{i}^{(W)}$ and $\hat{p}^{(W)}$ are time dependent Fourier amplitudes. The turbulent quantities far from the boundary, being homogeneous also in the vertical, may be expressed as Fourier integrals (with vertical wavenumber $k_{3}$ ) also along that direction.

The wavenumbers evolve in time under the action of the mean shear according to a 'conservation of wave crests' (Hunt 1973):

$$
\frac{\partial \vec{k}}{\partial t}+\nabla(\vec{k} \cdot \vec{U})=0
$$

where $\vec{k}=\left(k_{1}, k_{2}, k_{3}\right)$ is the three-dimensional wavenumber vector. The horizontal components of the wavenumber vector are then not affected by the mean shear, but the vertical component has a linear dependence on time (Townsend 1976):

$$
k_{1}(t)=k_{01}, \quad k_{2}(t)=k_{02} \quad \text { and } \quad k_{3}(t)=k_{03}-k_{01} \Gamma t
$$

where $\vec{k}_{0}=\vec{k}(t=0)=\left(k_{01}, k_{02}, k_{03}\right)$. For simplicity, the shorter notation $k_{1}$ and $k_{2}$ will be retained henceforth instead of $k_{01}$ and $k_{02}$.

\subsection{Evolution of the waves and wave velocity}

A vorticity equation is obtained by taking the curl of (5). If the curl is then taken of the vorticity equation we obtain an equation for $\nabla^{2} \vec{u}^{(W)}$. Since the there is only a simple shear in the mean flow, the vertical component of that equation takes a simple form: 


$$
\left(\frac{\partial}{\partial t}+U_{1}\left(x_{3}\right) \frac{\partial}{\partial x_{1}}\right)\left(\nabla^{2} u_{3}^{(W)}\right)=0 .
$$

The solution is

$$
\nabla^{2} u_{3}^{(W)}=G\left(x_{1}-U_{1}\left(x_{3}\right) t, x_{2}, x_{3}\right)
$$

where $G$ is a function determined by initial conditions. Since there are no waves initially, $u_{3}^{(W)}(t=0)=0, G=0$. Far from the interface the surface wave motion, $u_{3}^{(W)}$, decays to zero, whereas at the interface the boundary condition is (7). Using (9), the solution for $\hat{u}_{3}^{(W)}$ is then

$$
\hat{u}_{3}^{(W)}=\frac{\partial \hat{\zeta}}{\partial t} \mathrm{e}^{k_{12} x_{3}}
$$

where $k_{12}=\left(k_{1}^{2}+k_{2}^{2}\right)^{\frac{1}{2}}$. The solution for the vertical component of the wave velocity is the same as for irrotational waves; the solution for the horizontal components may not be.

In the case treated by Phillips (1957), when the mean shear and turbulence are in the air, the wave motion remains irrotational for all time. But when the mean shear and turbulence are in the water flow, the horizontal components of the wave motion become rotational over time. Hence the dynamical boundary condition is here developed more generally. The horizontal divergence of momentum equation of the wave motion (5) combined with mass conservation yields

$$
\left(\frac{\partial}{\partial t}+U_{1} \frac{\partial}{\partial x_{1}}\right) \frac{\partial u_{3}^{(W)}}{\partial x_{3}}-\Gamma \frac{\partial u_{3}^{(W)}}{\partial x_{1}}=\frac{1}{\rho_{w}} \nabla_{H}^{2} p^{(W)},
$$

where $\nabla_{H}^{2}=\partial / \partial x_{1}^{2}+\partial / \partial x_{2}^{2}$. This equation is evaluated at the interface, where $U_{1}=0$. It is then combined with the dynamical boundary condition that the discontinuity of the pressure across the interface is due only to surface tension. The two cases of turbulence in the water and turbulence in the air then need to be treated separately. 


\subsubsection{Turbulence in the water}

Since the waves are forced by the turbulence, but the interface is assumed stress-free, the turbulent pressure plus the wave-induced pressure at the interface is zero. The pressure in the linearised boundary condition (15) can be determined by evaluating the momentum equations in curvilinear coordinates at the interface, which yields

$$
p^{(W)}+p=\rho_{w}\left[g \zeta-\gamma \nabla_{H}^{2} \zeta\right] \quad \text { at } z=0
$$

where $g=9.8 \mathrm{~m} \mathrm{~s}^{-2}$ is the acceleration of gravity and $\gamma=72.6 \times 10^{-6} \mathrm{~m}^{3} \mathrm{~s}^{-2}$ is the surface tension, and the curvature term has been linearised.

Using (16), the dynamical boundary condition at $x_{3}=0,(15)$, becomes

$$
\frac{\partial}{\partial t}\left(\frac{\partial u_{3}^{(W)}}{\partial x_{3}}\right)-\Gamma \frac{\partial u_{3}^{(W)}}{\partial x_{1}}-g \nabla_{H}^{2} \zeta+\gamma \nabla_{H}^{4} \zeta=-\frac{1}{\rho_{w}} \nabla_{H}^{2} p
$$

Inserting the definitions of $u_{3}^{(W)}, \zeta$ and $p$ given by (9) into (17) and using also (14), it follows that,

$$
\frac{\partial^{2} \hat{\zeta}}{\partial t^{2}}-\mathrm{i} \Gamma \frac{k_{1}}{k_{12}} \frac{\partial \hat{\zeta}}{\partial t}+\sigma_{0}^{2} \hat{\zeta}=\frac{k_{12}}{\rho_{w}} \hat{p}\left(x_{3}=0\right)
$$

where

$$
\sigma_{0}^{2}=g k_{12}+\gamma k_{12}^{3}
$$

defines the natural angular frequency of surface waves in still water. The solution of (18) subject to the boundary conditions $\hat{\zeta}(t=0)=\partial \hat{\zeta} / \partial t(t=0)=0$ is

$$
\hat{\zeta}\left(k_{1}, k_{2}, t\right)=\frac{k_{12}}{\rho_{w} \sigma_{1}} \int_{0}^{t} \hat{p}\left(k_{1}, k_{2}, x_{3}=0, s\right) \mathrm{e}^{\mathrm{i} \frac{\Gamma k_{1}}{2 k_{12}}(t-s)} \sin \left[\sigma_{1}(t-s)\right] \mathrm{d} s,
$$


where $\sigma_{1}$ is defined as

$$
\sigma_{1}^{2}=\sigma_{0}^{2}\left[1+\left(\frac{\Gamma k_{1}}{2 k_{12} \sigma_{0}}\right)^{2}\right]
$$

The solution (20) is expressed as a time integral involving the pressure fluctuations at the air-water interface. The surface elevation is therefore determined at each instant by the history of these pressure fluctuations since the inception of the turbulent current.

\subsubsection{Turbulence in the air}

This is the case treated by Phillips (1957), and it is sufficient to note that since the wave motions begin from rest, they remain irrotational in this infinite Reynolds number limit. Then, the equation that the amplitude of the surface elevation must satisfy is obtained by simply setting $\Gamma=0$ in (18) and changing the sign of the turbulent pressure, which arises because the pressure acts on the other side of the interface. This leads to

$$
\frac{\partial^{2} \hat{\zeta}}{\partial t^{2}}+\sigma_{0}^{2} \hat{\zeta}=-\frac{k_{12}}{\rho_{w}} \hat{p}\left(x_{3}=0\right)
$$

This is equivalent to Phillips' (1957) equation (2.12). The solution of (22) for an initially unperturbed interface, where $\hat{\zeta}=\partial \hat{\zeta} / \partial t=0$ at $t=0$, is

$$
\hat{\zeta}\left(k_{1}, k_{2}, t\right)=-\frac{k_{12}}{\rho_{w} \sigma_{0}} \int_{0}^{t} \hat{p}\left(k_{1}, k_{2}, x_{3}=0, s\right) \sin \left[\sigma_{0}(t-s)\right] \mathrm{d} s .
$$

There are two differences between the solution for waves generated by mean shear and turbulence in the water, (20), and the solution for waves generated by mean shear and turbulence in the air, (23). Firstly, mean shear in the water changes slightly the dispersion relation of the surface waves, and secondly, the mean shear creates an impedance to surface waves, which yields the factor of $\exp \left[\left(\mathrm{i} \Gamma k_{1} / 2 k_{12}\right)(t-s)\right]$ in $(20)$. 


\subsection{Turbulence in shear flow near a boundary}

The expressions for the surface wave fields require the turbulent pressure fluctuations at the boundary. These are calculated from the turbulent velocity field in the RDT approximation, following Durbin (1978). A situation where the turbulence is below the boundary will be considered next as an example, but the treatment is analogous if the turbulence is above the boundary.

The linearised momentum equation that governs the turbulence velocity (4) is manipulated as was the equation for the wave velocity (5) to yield

$$
\left(\frac{\partial}{\partial t}+U_{1}\left(x_{3}\right) \frac{\partial}{\partial x_{1}}\right)\left(\nabla^{2} u_{3}\right)=0 .
$$

That equation has the solution

$$
\nabla^{2} u_{3}=F\left(x_{1}-U_{1}\left(x_{3}\right) t, x_{2}, x_{3}\right)
$$

where $F$ is an arbitrary function. But the similarities between the wave and turbulent motions end at this point. While the wave velocity is initially irrotational, the turbulent velocity is by definition rotational, so the function $F$ is not zero and is defined instead by the initial condition (cf. Durbin 1978)

$$
F(t=0)=\nabla^{2} u_{3}\left(x_{1}, x_{2}, x_{3}, t=0\right)
$$

The boundary and initial conditions simplify if it is recalled that the turbulence far from the boundary is assumed to be homogeneous (Durbin 1978). Since the shear rate $\Gamma$ is constant, the turbulence remains homogeneous at all times. Thus, the turbulent velocity far from the boundary can be expressed as a three-dimensional Fourier integral,

$$
u_{i}^{(H)}(\vec{x}, t)=\iiint \hat{u}_{i}^{(H)}(\vec{k}, t) \mathrm{e}^{\mathrm{i} \vec{k} \cdot \vec{x}} \mathrm{~d} k_{1} \mathrm{~d} k_{2} \mathrm{~d} k_{3},
$$


where $\hat{u}_{i}^{(H)}$ is the Fourier amplitude and, as seen previously, the wavenumber vector $\vec{k}$ is time dependent due to the shear, which makes the turbulence anisotropic over time. Towards the boundary, the turbulence is made inhomogeneous in the $x_{3}$ direction by the blocking effect. This effect can be taken into account by adding to $u_{i}^{(H)}$ a blocking correction which enables the flow to satisfy the boundary condition at the interface (first equation of (7)). Far from the boundary, as $x_{3} \rightarrow-\infty$, this blocking correction decays to zero. Following Durbin (1978), the initial condition states that the blocking correction is initially irrotational. Hence the solution for the velocity field at $t=0$ is identical to the corresponding solution, allowing for blocking, in the case of shear-free turbulence near a wall (Hunt and Graham 1978). In particular, the Fourier transform of the vertical velocity component takes the form

$$
\hat{u}_{3}\left(k_{1}, k_{2}, x_{3}, t=0\right)=\int \hat{u}_{3}^{(H)}\left(\vec{k}_{0}, t=0\right)\left(\mathrm{e}^{\mathrm{i} k_{03} x_{3}}-\mathrm{e}^{k_{12} x_{3}}\right) \mathrm{d} k_{03} .
$$

This expression can be introduced in (26) to obtain the form of the function $F\left(x_{1}, x_{2}, x_{3}\right)$. Replacing the argument $x_{1}$ of that function by $x_{1}-U_{1}\left(x_{3}\right) t$ and substituting the resulting expression in (25) yields an equation for $u_{3}$ in the general, time-dependent case. This equation is subject to the same boundary conditions as at $t=0$.

Accordingly, the time-dependent solution for the Fourier transform of the vertical velocity component is (Durbin 1978)

$$
\hat{u}_{3}\left(k_{1}, k_{2}, x_{3}, t\right)=\int \frac{k_{0}^{2}}{k^{2}} \hat{u}_{3}^{(H)}\left(\vec{k}_{0}, t=0\right)\left(\mathrm{e}^{\mathrm{i} k_{3} x_{3}}-\mathrm{e}^{k_{12} x_{3}}\right) \mathrm{d} k_{3},
$$

where $k_{0}=\left(k_{1}^{2}+k_{2}^{2}+k_{03}^{2}\right)^{\frac{1}{2}}$ and $k=\left(k_{1}^{2}+k_{2}^{2}+k_{3}^{2}(t)\right)^{\frac{1}{2}}$. For the case of turbulence in the air, only the sign of the exponent of the second exponential in (29) has to be changed, since the blocking effect decays exponentially upward instead of downward. The form of the horizontal velocity components $u_{1}$ and $u_{2}$ is rather more involved and has been obtained by, for example, Lee and Hunt (1989) 
and Mann (1994). However, for the problem of surface wave generation, only the pressure statistics are of interest, and in the RDT approximation these only depend on the vertical component of the turbulent velocity, as will be seen next.

\subsection{Pressure in the RDT approximation}

In Section 2.2, the wave amplitude was determined as a function of the amplitude of the turbulent pressure fluctuations at the air-water interface. The turbulent pressure field is now related to the turbulent velocity field calculated in Section 2.3 making use of the simplifying assumptions of RDT. Again, the case of turbulence in the water is considered as an example, but the treatment for turbulence in the air is entirely analogous.

Taking the divergence of (4), it follows that

$$
\nabla^{2} p=-2 \rho \Gamma \frac{\partial u_{3}}{\partial x_{1}}
$$

To obtain the turbulent pressure field everywhere, this equation has to be solved subject to the boundary conditions

$$
p\left(x_{3} \rightarrow-\infty\right)=p^{(H)} \quad \text { and } \quad \frac{\partial p}{\partial x_{3}}\left(x_{3}=0\right)=0,
$$

where $p^{(H)}(\vec{x}, t)$ is the pressure field associated with the turbulence far from the boundary, and where the boundary condition at $x_{3}=0$ was obtained from the vertical component of the momentum equation (4), taking into account the first equation of (7) (blocking condition). $p^{(H)}$ is defined as the solution of

$$
\nabla^{2} p^{(H)}=-2 \rho \Gamma \frac{\partial u_{3}^{(H)}}{\partial x_{1}} .
$$

Using (8), the Poisson equation (30) may be simplified, becoming an ordinary 
differential equation for the Fourier amplitudes of the pressure and velocity:

$$
\frac{\partial^{2} \hat{p}}{\partial x_{3}^{2}}-k_{12}^{2} \hat{p}=-2 \rho \Gamma \mathrm{i} k_{1} \hat{u}_{3}
$$

The solution for the Fourier transform of the turbulent velocity (29) may then be used, and the boundary conditions (31) applied.

After some algebra, the solution for the Fourier amplitude of the pressure is found to be

$$
\hat{p}=2 \mathrm{i} \rho \Gamma k_{1} \int \frac{k_{0}^{2}}{k^{2}} \hat{u}_{3}^{(H)}\left[\frac{1}{k^{2}} \mathrm{e}^{\mathrm{i} k_{3} x_{3}}+\left(\frac{x_{3}}{2 k_{12}}-\frac{1}{2 k_{12}^{2}}-\frac{\mathrm{i} k_{3}}{k_{12} k^{2}}\right) \mathrm{e}^{k_{12} x_{3}}\right] \mathrm{d} k_{3},
$$

which at the boundary takes the simplified form

$$
\hat{p}\left(k_{1}, k_{2}, x_{3}=0, t\right)=\mathrm{i} \rho \Gamma \frac{k_{1}}{k_{12}^{2}} \int \frac{k_{0}^{2}}{\left(\mathrm{i} k_{3}+k_{12}\right)^{2}} \hat{u}_{3}^{(H)}\left(k_{0}, t=0\right) \mathrm{d} k_{3},
$$

almost exactly as obtained by Durbin (1978). For turbulence in the water, (35) may be used directly, with $\rho$ replaced by $\rho_{w}$. For turbulence in the air, it is only necessary to change the sign of $k_{3}$ in (35) (because the pressure acts on the other side of the interface) and to replace $\rho$ by the density of air, $\rho_{a}$.

Since the turbulent pressure that drives the waves is now known, it remains to substitute the pressure amplitude (35) in (20) or its equivalent for turbulence in the air in (23) to obtain the resulting wave field as a function of the turbulent velocity field. It is then possible to calculate statistics of the waves.

\subsection{Statistics of the flow}

Since the present model is linear, the turbulent velocity and pressure, and the surface elevation can be related to the undistorted, initial, turbulence. Hence,

$$
u_{i}(\vec{x}, t)=\iiint M_{i j}\left(\vec{k}, x_{3}, t\right) \hat{u}_{j}^{(H)}\left(\vec{k}_{0}, t=0\right) \mathrm{e}^{\mathrm{i}\left(k_{1} x_{1}+k_{2} x_{2}\right)} \mathrm{d} k_{1} \mathrm{~d} k_{2} \mathrm{~d} k_{3},
$$




$$
\begin{aligned}
& p(\vec{x}, t)=\iiint Q_{j}\left(\vec{k}, x_{3}, t\right) \hat{u}_{j}^{(H)}\left(\vec{k}_{0}, t=0\right) \mathrm{e}^{\mathrm{i}\left(k_{1} x_{1}+k_{2} x_{2}\right)} \mathrm{d} k_{1} \mathrm{~d} k_{2} \mathrm{~d} k_{3}, \\
& \zeta\left(x_{1}, x_{2}, t\right)=\iiint S_{j}\left(\vec{k}, x_{3}, t\right) \hat{u}_{j}^{(H)}\left(\vec{k}_{0}, t=0\right) \mathrm{e}^{\mathrm{i}\left(k_{1} x_{1}+k_{2} x_{2}\right)} \mathrm{d} k_{1} \mathrm{~d} k_{2} \mathrm{~d} k_{3},
\end{aligned}
$$

where the matrix $M_{i j}$ and the vectors $Q_{j}$ for turbulence in the water and also $S_{j}$ are given in appendix A. A matrix akin to $M_{i j}$ was derived in the study of Mann (1994) where, however, there is a minor mistake which has been corrected in appendix A. The vector $Q_{j}$ may be obtained from (35). Finally, $S_{j}$ can be determined after substituting (35) into (20) or its equivalent for turbulence in the air into (23).

This paper focuses primarily on the statistics of the wave field induced by the turbulence. The two-dimensional wavenumber spectrum of the air-water interface elevation is defined as

$$
\begin{aligned}
& \Psi\left(k_{1}, k_{2}, t\right) \\
& =\frac{1}{(2 \pi)^{2}} \iint \overline{\zeta\left(x_{1}, x_{2}, t\right) \zeta\left(x_{1}+r_{1}, x_{2}+r_{2}, t\right)} \mathrm{e}^{-\mathrm{i}\left(k_{1} r_{1}+k_{2} r_{2}\right)} \mathrm{d} r_{1} \mathrm{~d} r_{2},
\end{aligned}
$$

where the overbar denotes ensemble averaging. Taking into account (36), this spectrum can be expressed as a function of the three-dimensional wavenumber spectrum of the undistorted turbulent velocity, $\Phi_{i j}^{(H)}$, in the following way (cf. Hunt 1973),

$$
\Psi\left(k_{1}, k_{2}, t\right)=\int S_{k}^{*} S_{m} \Phi_{k m}^{(H)}\left(\vec{k}_{0}\right) \mathrm{d} k_{3}
$$

where the asterisk denotes complex conjugate. The spectrum of the undistorted turbulent velocity can be related to the corresponding Fourier amplitudes, $\hat{u}_{i}^{(H)}$, using

$$
\overline{\hat{u}_{i}^{*(H)}\left(\vec{k}_{0}\right) \hat{u}_{j}^{(H)}\left(\vec{k}_{0}^{\prime}\right)}=\Phi_{i j}^{(H)}\left(\vec{k}_{0}\right) \delta\left(\vec{k}_{0}-\vec{k}_{0}^{\prime}\right) .
$$


If the undistorted turbulence is isotropic, this spectrum takes the form

$$
\Phi_{i j}^{(H)}\left(\vec{k}_{0}\right)=\left(\delta_{i j}-\frac{k_{0 i} k_{0 j}}{k_{0}^{2}}\right) \frac{E\left(k_{0}\right)}{4 \pi k_{0}^{2}},
$$

where $E\left(k_{0}\right)$ is the energy spectrum of the undistorted turbulence. Following Hunt and Graham (1978) and Durbin (1978), the well known von Kármán form of the energy spectrum, which has proved to be appropriate for inviscid calculations, is adopted here, namely

$$
E\left(k_{0}\right)=u^{2} l \frac{g_{2}\left(k_{0} l\right)^{4}}{\left(g_{1}+\left(k_{0} l\right)^{2}\right)^{\frac{17}{6}}}
$$

where $g_{1}=0.558$ and $g_{2}=1.196$ are dimensionless constants. $u$ and $l$, the velocity and length scales of the turbulence used in (41), are defined, respectively, as the initial root-mean-square (RMS) velocity and longitudinal integral length scale of the turbulence far from the boundary.

The surface elevation spectrum (38) is found to have a relatively simple explicit form, due to the fact that it only depends on the vertical component of the turbulent velocity. From (38), (40) and appendix A, this spectrum may be written

$$
\Psi\left(k_{1}, k_{2}, t\right)=\frac{1}{4 \pi} \frac{\rho_{a}^{2}}{\rho_{w}^{2}} \Gamma^{2} \frac{k_{1}^{2}}{\sigma_{0}^{2}} \int E\left(k_{0}\right)\left|\int_{0}^{t} \frac{\sin \left(\sigma_{0}(t-s)\right)}{\left(k_{12}+\mathrm{i} k_{3}(s)\right)^{2}} \mathrm{~d} s\right|^{2} d k_{03},
$$

for turbulence in the air, while for turbulence in the water, it has the form

$$
\begin{aligned}
& \Psi\left(k_{1}, k_{2}, t\right) \\
& =\frac{1}{4 \pi} \Gamma^{2} \frac{k_{1}^{2}}{\sigma_{1}^{2}} \int E\left(k_{0}\right)\left|\int_{0}^{t} \frac{\mathrm{e}^{\mathrm{i} \frac{\Gamma k_{1}}{2 k_{12}} s}}{\left(k_{12}-\mathrm{i} k_{3}(s)\right)^{2}} \sin \left(\sigma_{1}(t-s)\right) \mathrm{d} s\right|^{2} d k_{03} .
\end{aligned}
$$

The expressions between brackets in (42) and (43) can be expanded into an oscillating part and a growing part (see appendix B). The oscillating part, although formally accurate, corresponds to the addition of an oscillatory function of time and wavenumber to the spectra, which prevents the statistics 
from being smooth. For that reason, in the following numerical calculations, only the growing part of the solution will be considered. This is approximately equivalent to time averaging the spectra over an interval sufficiently long to filter the oscillations, but shorter than the time scale relevant for wave growth. This filtering does not correspond to a neglect of the non-resonant waves. It is also found that the time integrals in the expressions between brackets can be written explicitly in terms of exponential integral functions of a complex argument. The final form taken by these expressions is rather lengthy, so it has been left for appendix C.

It can be seen from (42) and (43) that the integrals between brackets are somewhat akin to a Fourier transformation in the time domain. Over a sufficiently long interval, the factors $\sin \left(\sigma_{0}(t-s)\right)$ and $\exp \left[\left(\mathrm{i} \Gamma k_{1} / 2 k_{12}\right) s\right] \sin \left(\sigma_{1}(t-s)\right)$ select frequencies of the forcing which are close to the resonance frequency, thus making the surface wave spectrum grow preferentially for the combination of frequency and wavenumber that corresponds to freely-propagating waves (if a pressure forcing exists at that frequency). The time-dependent expression in the denominator, however, increases in time and limits this growth. Physically, this increase is associated with the progressive elongation of the streaky structures in the turbulence (Lee et al. 1990) as the total distortion imposed on the turbulence by the shear becomes larger. Ultimately, this distortion is limited by the second condition of (3), when the boundary layer reaches an equilibrium state.

This situation differs from that considered by Phillips (1957) in that the statistics of the turbulent pressure are not stationary. If the initial phase of surface wave growth happens over a reasonably short time scale (as seems to be supported by experiment), the air or water boundary layer does not have the time to achieve a steady state from the inception of the flow to the instant when feedback wave amplification mechanisms become dominant. So, the type of unsteadiness considered in the present model, with departure of the turbulence 
from an isotropic initial state, and its subsequent distortion by a constant mean shear may be regarded as a reasonable leading-order approximation to a developing boundary layer.

\subsection{Dimensionless parameters}

In order to avoid redundancy, it is convenient to reduce the number of input variables of the model to a minimum. This can be done by making all quantities dimensionless, using $l$ and $u$ (e.g. $\Gamma^{\prime}=\Gamma l / u$ ), except for the dimensionless time, which is defined as $t^{\prime}=\Gamma t$. Dimensionless variables are hereafter denoted by a prime.

It can be shown that the parameters controlling the dimensionless wave elevation spectrum $\Psi^{\prime}$ and other related quantities, are $k_{12}^{\prime}, t^{\prime}$ and the Froude and Weber numbers of the turbulence,

$$
F r=\frac{u}{(g l)^{\frac{1}{2}}}, \quad W e=\frac{l u^{2}}{\gamma}
$$

$F r$ and $W e$ estimate the relative importance of the forcing at the interface due to the turbulence and the restoring forces due to gravity and surface tension, respectively. The larger these two numbers are, the easier it becomes for the interface to be deformed by the turbulence (Brocchini and Peregrine 2001).

Since $g$ and $\gamma$ are fixed physical constants, (44) implies that $F r$ and $W e$ are uniquely related to $l$ and $u$ and either pair of variables may be used interchangeably. As in Brocchini and Peregrine (2001), $l$ and $u$ will be adopted instead of $\mathrm{Fr}$ and $\mathrm{We}$ as direct input parameters to the present surface wave model, because physically plausible values are more readily found for these dimensional quantities. The use of $F r$ and $W e$ would perhaps be more appropriate in situations where the fluids under consideration are not, specifically, air and water. 


\section{Results}

Results are now presented for turbulence in either the water or air. First the magnitude of the pressure fluctuations needs to be decided. Since the pressure variance in boundary layer flows is proportional to the shear stress (Bull 1996) and the shear stress is continuous across the interface in coupled air-water flows (Kondo 1976), it will be assumed in what follows that the pressure fluctuations for turbulence in the water and turbulence in the air have the same magnitude. As mentioned above, the input parameters of the present model are $l, u, t^{\prime}$ and $\Gamma^{\prime}$. The relation between these parameters that ensures that this condition is satisfied is defined next.

The pressure variance at the interface can be obtained by squaring the second equation of (36) and taking the ensemble average, using the expressions for $Q_{i}$ available in appendix A (or the equivalent expressions for turbulence in the air) and also (40). After some algebra, the dimensionless pressure variance is found to be:

$$
\overline{p^{\prime 2}}\left(x_{3}^{\prime}=0, t^{\prime}\right)=\frac{1}{4 \pi} \iiint \frac{k_{1}^{\prime 2}}{k_{12}^{\prime 2}} \frac{E^{\prime}\left(k_{0}^{\prime}\right)}{k^{\prime 4}\left(t^{\prime}\right)} \mathrm{d} k_{1}^{\prime} \mathrm{d} k_{2}^{\prime} \mathrm{d} k_{3}^{\prime},
$$

where

$$
p^{\prime}=\frac{p}{\rho \Gamma u l}
$$

By inspection of (45), it is clear that the dimensionless pressure variance at the interface is only a function of $t^{\prime}$. Hence, for a given $t^{\prime}$, the dimensional pressure variance is proportional to $(\rho \Gamma u l)^{2}$. This reasoning is valid both for turbulence in the water and for turbulence in the air. So, in order to have pressure fluctuations of a similar magnitude in both cases, the product $\Gamma u l$ must be larger in the air than in the water by a factor $\rho_{w} / \rho_{a}$. In the following treatment, $\rho_{w} / \rho_{a}=10^{3}$ is assumed and, to satisfy the above constraint in a 
very simple way, $\Gamma, u$ and $l$ are each taken to be larger in the air than in the water by a factor of 10 . Although rough, this estimate is in qualitative agreement with experiment: it is well known that shear is more intense and turbulence more vigorous and characterised by larger eddies in the atmosphere than in the ocean (Donelan 1990). The values of $u$ and $l$ used in the model for turbulence in the water, of the order of a few centimetres per second and centimetres, respectively, seem reasonable for oceanic turbulence (cf. Fig. 4 of Caulliez et al. 1998, and Kitaigorodskii et al. 1983). The dimensionless shear rate is taken to be 10, a value which is supported by numerical experiments of flows, albeit at relatively low Reynolds numbers (Lee et al. 1990, cf. their Fig. 1). Additionally, noting that the dominant contributions to the pressure at a flat boundary come from within the so-called buffer layer, at a distance from the boundary $z \approx 20 \nu / u_{*}$ (Chang et al. 1999), the shear rate may be estimated at that distance, as $\Gamma \approx u_{*}^{2} /(20 \kappa \nu)$, based on a logarithmic profile. Using typical values for turbulence in the water, such as $u_{*}=2.0 \mathrm{~cm} \mathrm{~s}^{-1}$ and $\nu=1.0 \times 10^{-6} \mathrm{~m}^{2} \mathrm{~s}^{-1}$ yields $\Gamma=10 \mathrm{~s}^{-1}$, which for the values of $u$ and $l$ quoted above also corresponds to $\Gamma^{\prime} \approx 10$.

\subsection{Mean square slope of the waves}

The mean square slope (MSS) of the surface waves generated at the air-water interface will be determined first, because it gives a single measure of the waves and also of their likeliness to be affected by the feedback generation mechanisms - the pressure fluctuations associated with these mechanisms are proportional to the wave slope (Belcher and Hunt 1993). It should be emphasised that, since both gravity and surface tension were taken into account in the treatment of Section 2, the surface waves under consideration are gravitycapillary waves. The MSS can be found by integrating the surface elevation 
spectrum multiplied by $k_{12}^{2}$ over all wavenumbers:

$$
\overline{(\nabla \zeta)^{2}}=\overline{\left(\frac{\partial \zeta}{\partial x_{1}}\right)^{2}}+\overline{\left(\frac{\partial \zeta}{\partial x_{2}}\right)^{2}}=\iint k_{12}^{2} \Psi\left(k_{1}, k_{2}, t\right) \mathrm{d} k_{1} \mathrm{~d} k_{2}
$$

Fig. 2 shows the evolution of the MSS of the surface waves predicted by the model for turbulence in the water as a function of dimensionless time $t^{\prime}$. In Fig. 2a, the sensitivity of the MSS to $\Gamma^{\prime}$, keeping $l$ and $u$ constant, is tested. It can be seen that the wave growth is faster and more sustained for higher values of $\Gamma^{\prime}$. This was to be expected since, as was shown in the previous subsection, the pressure fluctuations that generate the waves are proportional to $\Gamma$.

In Fig. 2b, the MSS is plotted for different values of $u$, keeping $\Gamma^{\prime}$ and $l$ constant. It is observed that the waves grow faster when $u$ is higher. This is not surprising either, since the pressure fluctuations that drive the waves are proportional to $u$. There is an indirect effect which further promotes wave growth: when $\Gamma^{\prime}$ is kept constant and $u$ is increased, $\Gamma$ also increases proportionally. Because of this, the MSS is very sensitive to $u$.

Finally, Fig. 2c displays the variation of the MSS growth with $l$, keeping $\Gamma^{\prime}$ and $u$ constant. It can be seen that the waves grow faster for lower values of $l$. While the pressure fluctuations that generate the waves are proportional to $l, \Gamma$ decreases as $l$ increases when $\Gamma^{\prime}$ is kept constant and the two effects cancel. However, since the dominant wavelength of the generated waves is approximately equal to the length scale of the turbulence $l$, waves of a given amplitude tend to be steeper for smaller $l$.

With the physically reasonable values of $u, l$ and $\Gamma^{\prime}$ chosen, the model is able to produce waves with a RMS slope of $O(0.1)$ in the time interval considered. For example, when $l=5 \mathrm{~cm}, u=5 \mathrm{cms}^{-1}$ and $\Gamma^{\prime}=10$ (i.e. $\Gamma=10 \mathrm{~s}^{-1}$ ), the MSS takes a value of 0.002 at $t^{\prime}=3$ (which is within the range of total distortions used by Townsend 1976 or Lee et al. 1990). This means that the 
root-mean-square $(\mathrm{RMS})$ slope takes a value of $\approx 0.05$ at $0.3 \mathrm{~s}$. Having in mind the value of $l$, this corresponds to waves of $\approx 2.5 \mathrm{~mm}$ amplitude, which are of the same order of magnitude as the first 'visible' waves measured by Caulliez et al. (1998).

Fig. 3 shows plots of the MSS as a function of $t^{\prime}$ for the case of turbulence in the air, for values of $\Gamma^{\prime}, u$ and $l$ ten times larger than those used in Fig. 2, so as to produce pressure fluctuations of the same magnitude. Two differences immediately stand out between Fig. 2 and 3. Firstly, for turbulence in the air, the values of MSS attained are much lower than for turbulence in the water, despite the turbulent pressure fluctuations being of the same magnitude. In all the three plots in Fig. 3a-c, the MSS barely reaches $1 \times 10^{-6}$, which corresponds to a RMS slope of $1 \times 10^{-3}$. In real situations, waves with such a slope would be invisible to the naked eye and they appear too small to be amplified by feedback mechanisms in a reasonable time interval. This suggests that turbulence in the water may have a much more important role in the initial generation of surface waves than is generally believed.

Secondly, although the trends in the behaviour of wave growth with the parameters $\Gamma^{\prime}, u$ and $l$ are in the same sense as for turbulence in the water, the sensitivity to the shear rate $\Gamma^{\prime}$ is much reduced (see Fig. 3a. Both these aspects are linked with the decorrelation process of the turbulent pressure fluctuations over time, and will be explained in the next subsection.

The general appearance of the MSS curves in Fig. 2 and 3 is quite similar. In both cases the MSS initially increases at a fast rate, but later increases progressively more slowly, reaching an approximate plateau. This behaviour is in contrast with the prediction of linear growth by Phillips (1957), and is a consequence of the fact that here the evolution of the turbulent pressure fluctuations is modelled explicitly. This aspect will also be analysed in more detail in the next subsection. 


\subsection{Decorrelation of the pressure fluctuations}

To understand why the waves generated by turbulence in the air are much gentler than those generated by turbulence in the water despite the fact that the magnitude of the forcing pressure fluctuations is the same, recall that the spatio-temporal structure of these pressure fluctuations is also crucial for wave growth. Phillips (1957) pointed out that resonant wave growth occurs when pressure fluctuations advected over the air-water interface match both the wavenumber and the frequency of free surface waves. This resonance process would be maximised if the pressure fluctuations with the appropriate 'dispersion relation' were rigidly advected, following the waves with which they are able to resonate. But such perfect resonance never happens in turbulent flows. The interactions between the mean flow and the turbulence, or the turbulence with itself, mean that pressure patterns with given initial length and time scales are distorted by the velocity field, and are only capable of interacting resonantly with a wave for a finite time. That time is the decorrelation time of pressure, which Phillips calls $\theta$. Here we have calculated explicitly the decorrelation of pressure as the turbulence interacts with the mean shear flow. This is sometimes called the 'fast' part of the turbulent pressure. Decorrelation through this mechanism is particularly clear: shear is responsible for the variation of velocity with distance from the boundary, so that different sources of the pressure travel at different speeds and thus lose coherence.

A function that contains useful information about the decorrelation time of pressure is the temporal covariance function of the turbulent pressure fluctuations. That function can be determined at the air-water interface by multiplying the second equation of (8) at $x_{3}=0$ and at time $t$ by the same expression at time $t+\Delta t$ (where $\Delta t$ is a time lag), using the expressions of $Q_{j}$ in appendix A and ensemble averaging. The resulting expression can then be made dimensionless, yielding 


$$
\begin{aligned}
& \overline{p^{\prime}\left(t^{\prime}\right) p^{\prime}\left(t^{\prime}+\Delta t^{\prime}\right)} \\
& =\frac{1}{4 \pi} \iiint \frac{k_{1}^{\prime 2}}{k_{12}^{\prime 2}} \frac{E^{\prime}\left(k_{0}^{\prime}\right)}{\left(k_{12}^{\prime}-\mathrm{i} k_{3}^{\prime}\left(t^{\prime}\right)\right)^{2}\left(k_{12}^{\prime}+\mathrm{i} k_{3}^{\prime}\left(t^{\prime}+\Delta t^{\prime}\right)\right)^{2}} \mathrm{~d} k_{1}^{\prime} \mathrm{d} k_{2}^{\prime} \mathrm{d} k_{3}^{\prime},
\end{aligned}
$$

where $\Delta t^{\prime}=\Gamma \Delta t$ is the dimensionless time lag. The dimensionless pressure covariance only depends on $t^{\prime}$ and $\Delta t^{\prime}$. If this covariance is divided by the dimensionless pressure variance (45), the pressure correlation function is obtained. The pressure correlation has been plotted in Fig. 4 for $t^{\prime}=0,3$ and 5 , as a function of $\Delta t^{\prime}$. It can be seen that the dependence of the correlation function on $t^{\prime}$ is relatively weak. Concerning the dependence on $\Delta t^{\prime}$, the pressure correlation has a maximum at the origin, then becomes slightly negative, and for large values of $\Delta t^{\prime}$ decays to zero. The point where this function intercepts the horizontal axis remains approximately the same for every $t^{\prime}$. Now, the location of this intercept can be understood as giving a possible definition for the decorrelation time of pressure. In Fig. 4, the intercept corresponds to a value of $\Delta t^{\prime} \approx 1.5$. So, it can be concluded that the dimensional decorrelation time for pressure is $\Delta t_{D} \approx 1.5 / \Gamma$. Hence the turbulent pressure fluctuations remain coherent for a shorter time as the shear rate increases, which seems intuitive.

Since the shear rate is higher in the air than in the water, the decorrelation time of the turbulent pressure fluctuations is shorter in the air than in the water. For wave generation purposes, this effect counteracts the proportionality of the pressure to the shear rate which was seen to exist at the beginning of Section 3. For, even if the pressure fluctuations in the air have the same magnitude as those in the water, they are considerably less coherent, and that is one of the reasons for the reduced growth of the MSS for turbulence in the air (Fig. 3).

The reason why the wave growth depends very weakly on $\Gamma^{\prime}$ in the case of turbulence in the air (Fig. 3a) is that the opposing effects of increasing $\Gamma$ on the magnitude of the pressure fluctuations and on their decorrelation time almost 
offset each other for high values of $\Gamma^{\prime}$. This cancellation does not occur for turbulence in the water (Fig. 2a) because at the lowest values of $\Gamma$ employed in that case there is no resonant growth, and the coherence of the pressure fluctuations (reflected in the integral time scale) is only important for resonant wave growth.

That the lowest curves in Fig. 2a correspond to non-resonant waves is shown by the fact that the MSS stabilizes to a constant value very early. This may also be noted in Fig. 2b for the lowest values of $u$ and in Fig. 2c for the highest values of $l$. In contrast, for turbulence in the air (Fig. 3) the slope of all the curves decreases as $t^{\prime}$ increases but never quite becomes zero. The lowest curves in Fig. 2, corresponding to non-resonant waves generated by turbulence in the water, although distinctly gentler than resonant waves generated by turbulence in the water, are still considerably steeper than resonant waves generated by turbulence in the air. This result further supports the view that turbulence in the water is important in wave growth.

While a constant-shear flow such as that employed in the present study would, in principle, always be able to generate resonant waves, because its mean velocity increases without bound away from the boundary, in the cases mentioned above the shear rate $\Gamma$ is sufficiently small that the flow velocity only reaches the minimum phase speed of surface waves at a distance from the boundary for which it gives a very small contribution to the surface pressure. This explains the observed disappearance of resonant waves at low $\Gamma$.

A qualitative feature of Fig. 2 and 3 that was noted in Section 3.1 is the slowing down in the growth rate of the MSS as time progresses, even in conditions thought to be resonant. That feature can be understood by analysing the time evolution of the turbulent pressure fluctuation statistics. The onedimensional wavenumber spectrum of the pressure in the streamwise direction 
at the interface can be shown to have the form

$$
\Pi_{1}\left(k_{1}, x_{3}=0, t\right)=\iint Q_{k}^{*} Q_{m}\left(x_{3}=0\right) \Phi_{k m}^{(H)}\left(\vec{k}_{0}\right) \mathrm{d} k_{2} \mathrm{~d} k_{3}
$$

Using the expressions for $Q_{k}$ from appendix A and also (40), (49) can be simplified and made dimensionless, yielding

$$
\Pi_{1}^{\prime}\left(k_{1}^{\prime}, x_{3}^{\prime}=0, t^{\prime}\right)=\frac{1}{4 \pi} \iint \frac{k_{1}^{\prime 2}}{k_{12}^{\prime 2}} \frac{E^{\prime}\left(k_{0}^{\prime}\right)}{k^{\prime 4}\left(t^{\prime}\right)} \mathrm{d} k_{2}^{\prime} \mathrm{d} k_{3}^{\prime},
$$

where $\Pi_{1}^{\prime}=\Pi_{1} /\left(\rho^{2} \Gamma^{2} u^{2} l^{3}\right)$. The dimensionless pressure spectrum only depends on $k_{1}^{\prime}$ and $t^{\prime}$ and is plotted in Fig. 5 for different values of $t^{\prime}$. As time advances, the peak in the spectrum moves towards lower wavenumbers and the energy at high wavenumbers decreases. This process corresponds to the elongation of the turbulent eddies in the streamwise direction as the total shear imposed on the turbulence increases, forming streaky structures. Such streaky structures have been observed, for example, in the numerical simulations of Lee et al. (1990) and in the wave initiation experiments of Caulliez et al. (1998).

Therefore, the pressure forcing at the interface moves towards larger scales. These scales are less susceptible to excitation and also contribute less to the surface wave slope, making the slope grow progressively more slowly. Although, in real cases, after some time the turbulence presumably reaches some type of equilibrium at an elongated 'streaky' state, the present results suggest that these streaks are considerably less efficient in generating waves than the more isotropic turbulence existing initially.

\subsection{Surface wave spectra}

Since the present model supports the idea that turbulence in the water is important in initially driving the surface waves, more detailed statistics of these waves are now presented. 
The curvature spectrum of the surface waves is defined as

$$
B\left(k_{1}, k_{2}, t\right)=k_{12}^{4} \Psi\left(k_{1}, k_{2}, t\right) .
$$

This spectrum is dimensionless by definition, and will be plotted next in the functional form $B\left(k_{12}, \theta, t^{\prime}\right)$, where $\theta$ is the angle of the wavenumber vector with the direction of the current.

Fig. 6 shows the sensitivity of the predicted curvature spectrum to the input variables $\Gamma^{\prime}, l$ and $u$, for an angle $\theta=0$ and a dimensionless time $t^{\prime}=5$. This time was chosen because, on the one hand, it is within the range of realistic total distortions (see last equation of (3)) and on the other corresponds approximately to the end of the stage of fastest MSS growth (see Fig. 2 ). The spectrum does not change appreciably for $t^{\prime}>5$, increasing only slightly at low wavenumbers. The spectrum is plotted as a function of the dimensional wavenumber rather than of the dimensionless wavenumber, because this bears out more clearly the important wavenumber $k_{\min }=367 \mathrm{~m}^{-1}$. This wavenumber corresponds to the transition between the gravity and the capillary wave regimes, where the phase speed of surface waves is a minimum, $c_{\text {min }} \approx 23 \mathrm{~cm} \mathrm{~s}^{-1}$.

The first aspect which stands out in Fig. 6a-c is that the curvature spectra of the waves are sharply peaked. The peak occurs roughly at the wavenumber $k_{12} \approx 2 \pi / l$. Some departure from this value towards lower wavenumbers can be attributed to the elongation that the turbulent eddies have suffered in the streamwise direction for $t^{\prime} \leq 5$ (cf. Fig. 5). Away from the peak, the spectra appear to vary proportionally to powers of the wavenumber, as shown by the straight portions of the curves. These power laws result directly from the assumed spectrum of the turbulent velocity. There is a breakpoint in the wave spectra at the wavenumber $k_{\text {min }}$.

In Fig. 6a, it can be seen that, as $\Gamma^{\prime}$ increases, the level of $B\left(k_{12}, 0\right)$ generally 
increases, in accordance with Fig. 2. The peak of the spectrum moves to the right, towards $k_{\text {min }}$, and the zone to the left of the peak lowers slightly, while the zone to the right rises. This happens because, for a higher $\Gamma$, the pressure decorrelation time is shorter, and hence the turbulence is only able to interact appreciably with the resonant waves of higher $\sigma_{0}$, and thus of higher $k_{12}$.

Fig. $6 \mathrm{~b}$ presents the dependence of the curvature spectrum on the turbulence RMS velocity $u$. The spectral density increases with $u$ everywhere, but more so at the peak and to the right of the peak. There is a slight drift of the peak towards higher wavenumbers as $u$ increases, but much less pronounced than in Fig. 6a.

Finally, Fig. 6c shows the dependence of $B\left(k_{12}, 0\right)$ on the turbulence length scale $l$. There is a general decrease on the values of the spectrum as the length scale increases, in accordance with the MSS results. The spectral peak moves towards lower wavenumbers when $l$ increases, as expected.

\subsection{Angular energy distribution}

The curvature spectrum contains information about the angular distribution of the wave energy, which is analysed next.

Fig. 7 displays plots of $B\left(k_{12}, \theta\right)$, for various values of $k_{12}$, as a function of $\theta$, at a dimensionless time $t^{\prime}=5$. In Fig. 7a, the spectral density distribution varies with the angle approximately as $\cos ^{2} \theta$, because of the factor $k_{1}^{2}$ present in the expression of the wave spectrum (43). This is a type of dependence often assumed in surface wave spectrum models (Phillips 1985) because it roughly fits observations. However, at the lowest wavenumber considered a slight broadening of the angular distribution can be noticed. In Fig. 7b, where the shear rate has been increased, a different kind of behaviour can be observed. While at the highest wavenumber, the spectral density distribution is still peaked at $\theta=0$, 
at lower wavenumbers the distribution becomes flattened and at even lower wavenumbers bimodal, with two peaks symmetrically placed about $\theta=0$.

This broadening of the spectrum occurs in the present model because the pressure fluctuations at the interface move at different speeds according to their scale. As noted by Durbin (1978), large-scale pressure fluctuations are induced by large turbulent eddies which exist at a relatively large distance from the interface, and which are advected at relatively high speed. Hence, these pressure fluctuations are also advected at relatively high speed (higher than $c_{\text {min }}$ in this case) and generate resonant waves propagating at an angle to the mean flow. The small-scale pressure fluctuations, on the contrary, are induced by small turbulent eddies at short distances from the interface, which all have a low advection velocity. Hence, these pressure fluctuations also move slowly (more slowly than $c_{m i n}$ ) and are unable to generate resonant waves. But the direction where the resonant condition is closest to being satisfied is that of the mean flow $(\theta=0)$.

The spectrum also broadens as $\Gamma^{\prime}$ increases, because a higher shear rate promotes larger advection velocities in the vicinity of the interface, thereby widening the range of $\theta$ at which resonance can occur. Note how the curves for the lower shear rate in Fig. 7a correspond to a MSS that stabilizes approximately in Fig. 2a while the curves for the higher shear rate in Fig. 7b correspond to a MSS that keeps on growing, although slowing down somewhat. This shows that both the absence of sustained growth and an angular energy distribution that strongly peaks at $\theta=0$ identify non-resonant waves.

In qualitative terms, the bimodal distribution of the wave spectrum for the case presented in Fig. 7b could have been predicted from Phillips' (1957) theory on the basis of these resonance arguments. However, the quantitative way in which the wave spectrum broadens as the wavenumber decreases is a result of the turbulence model adopted here for relating the turbulent pressure 
field and the turbulent velocity field. The existence of waves for wind speeds below $c_{\min }$ and angles larger than the resonance angle would not be predicted by Phillips' theory. But, as seen before, these forced, or non-resonant, waves may be relevant in real situations.

\section{Conclusions}

A theoretical study has been conducted about the initial stage of surface wave generation at an air-water interface by a weakly-turbulent constant-shear flow. The problems of turbulence in the air and of turbulence in the water have been addressed separately, so as to isolate the corresponding dynamical processes. Unlike previous studies, both resonant and non-resonant waves have been considered. The model developed in this study can be viewed as a combination between the wave generation theory of Phillips (1957) and the RDT analysis of Durbin (1978). It thus represents the early time evolution of the turbulent pressure fluctuations that generate the waves in a dynamically consistent way, enabling a calculation of the statistics of the turbulent pressure necessary in Phillips theory from simple parameters of the turbulent velocity field.

The mean-square-slope and the curvature spectrum of the surface waves are calculated as a function of time for turbulent pressure fluctuations of similar magnitude driven by turbulence in the water and in the air. It is found that the MSS increases faster for higher values of the dimensionless shear rate $\Gamma^{\prime}$ and initial RMS turbulent velocity $u$ and lower values of the initial integral length scale of the turbulence $l$. This is explained by the proportionality of the pressure applied at the interface to $\Gamma$ and $u$, and by the inverse proportionality of the wave slope to $l$. For resonant waves, the MSS growth rate does not depend appreciably on $\Gamma^{\prime}$, because the effect that the integral time scale of

the turbulence is inversely proportional to $\Gamma$ cancels with the effect that the 
pressure magnitude is proportional to $\Gamma$.

The waves generated by turbulence in the air are much gentler than the waves generated by turbulence in the water. This difference is particularly marked for resonant conditions, where this behaviour is attributed primarily to the fact that the integral time scale of turbulence in the water is larger and the length scale of that turbulence is smaller than that of turbulence in the air. For non-resonant waves, the integral time scale of the turbulence ceases to be a relevant parameter, and the magnitude of the MSS decreases by an order of magnitude approximately. However, the results suggest that even in that case the effect of turbulence in the water in the generation of surface waves may be dominant.

Wave curvature spectra are plotted for discrete wavenumbers, as a function of the angle between the direction of wave propagation and the direction of the mean flow, $\theta$. The spectra are found to peak like $\cos ^{2} \theta$ at $\theta=0$ for all wavenumbers at low shear rates, but to peak at $\theta=0$ for higher shear rates only at high wavenumbers, having a broader or even bimodal distribution at lower wavenumbers. This behaviour is found to be linked with the spatiotemporal structure of the pressure fluctuations, as modelled by RDT. Longer, resonant waves are excited by pressure fluctuations associated with larger and faster-moving turbulent eddies being advected at some distance from the boundary, while shorter waves grow due to the pressure fluctuations associated with smaller and slower-moving eddies, which exist closer to the boundary.

The main conclusion from this study is that turbulence in the water may be much more important for the initiation of surface waves than previously expected, because the associated pressure fluctuations are more efficient. The model suggests that the key stage in surface wave initiation may be that immediately following laminar-turbulent transition of the flow, when the turbulence has not become too anisotropic. The subsequent formation of streaky struc- 
tures due to shear distortion considerably slows down the wave growth, since the turbulent pressure forces the waves at progressively lower wavenumbers. These results are consistent with recent laboratory experiments by Caulliez et al. (1998) which support the idea of an explosive wave growth after the transition to turbulence of the shear current induced by the wind, and report the formation of turbulent streaks in that current.

It might be argued that the waves that are amplified by feedback instability mechanisms must be free waves, while the waves generated by a Phillips-type mechanism due to turbulence in the water are almost invariably forced waves, because the velocity of the shear current is almost always lower than the minimum phase speed of free waves. But these forced waves may establish the initial conditions for the existence of free waves of similar amplitude and wavelength, some of which may propagate approximately along the wind direction, and thus be in suitable conditions for being amplified by the feedback mechanisms investigated by Miles (1957) and Belcher and Hunt (1993).

\section{Acknowledgements}

The authors are grateful to Jakob Mann for assistance with the constant-shear RDT and also for providing a copy of his $\mathrm{PhD}$ thesis. M.A.C.T. acknowledges the financial support of the Portuguese Science Foundation (FCT), which funded this work under grant SFRH/BPD/3533/2000 and projects BULET/33980/99 and BOSS/58932/2004.

\section{Appendix A. Expressions of $M_{i j}, Q_{j}$ and $S_{j}$}

For turbulence in the water, the $M_{i j}$ matrix can be written 


$$
\begin{aligned}
& M_{11}=\mathrm{e}^{\mathrm{i} k_{3} x_{3}}, \\
& M_{12}=0, \\
& M_{13}=\mathrm{e}^{\mathrm{i} k_{3} x_{3}}\left\{\left(\frac{k_{1} k_{30}}{k_{12}^{2}}-\frac{k_{1} k_{3}}{k_{12}^{2}} \frac{k_{0}^{2}}{k^{2}}\right)-\frac{k_{2}^{2} k_{0}^{2}}{k_{1} k_{12}^{3}}\left\{\arctan \left(\frac{k_{30}}{k_{12}}\right)\right.\right. \\
& -\arctan \left(\frac{k_{3}}{k_{12}}\right)+\frac{1}{2 \mathrm{i}}\left[\mathrm { e } ^ { 2 k _ { 1 2 } x _ { 3 } } \left[E_{1}\left(\left(k_{12}+\mathrm{i} k_{30}\right) x_{3}\right)-E_{1}\left(\left(k_{12}+\mathrm{i} k_{3}\right) x_{3}\right)\right.\right. \\
& \left.+\pi \mathrm{i}\left(\operatorname{sign}\left(k_{3}\right)-\operatorname{sign}\left(k_{30}\right)\right)\right]-\left[E_{1}\left(\left(-k_{12}+\mathrm{i} k_{30}\right) x_{3}\right)\right. \\
& \left.\left.\left.\left.-E_{1}\left(\left(-k_{12}+\mathrm{i} k_{3}\right) x_{3}\right)\right]\right]\right\}\right\}-\mathrm{ie}^{k_{12} x_{3}} \frac{k_{1} k_{0}^{2}}{k_{12} k^{2}}, \\
& M_{21}=0, \\
& M_{22}=\mathrm{e}^{\mathrm{i} k_{3} x_{3}}, \\
& M_{23}=\mathrm{e}^{\mathrm{i} k_{3} x_{3}}\left\{\left(\frac{k_{2} k_{30}}{k_{12}^{2}}-\frac{k_{2} k_{3}}{k_{12}^{2}} \frac{k_{0}^{2}}{k^{2}}\right)+\frac{k_{2} k_{0}^{2}}{k_{12}^{3}}\left\{\arctan \left(\frac{k_{30}}{k_{12}}\right)\right.\right. \\
& -\arctan \left(\frac{k_{3}}{k_{12}}\right)+\frac{1}{2 \mathrm{i}}\left[\mathrm { e } ^ { 2 k _ { 1 2 } x _ { 3 } } \left[E_{1}\left(\left(k_{12}+\mathrm{i} k_{30}\right) x_{3}\right)-E_{1}\left(\left(k_{12}+\mathrm{i} k_{3}\right) x_{3}\right)\right.\right. \\
& \left.+\pi \mathrm{i}\left(\operatorname{sign}\left(k_{3}\right)-\operatorname{sign}\left(k_{30}\right)\right)\right]-\left[E_{1}\left(\left(-k_{12}+\mathrm{i} k_{30}\right) x_{3}\right)\right. \\
& \left.\left.\left.\left.-E_{1}\left(\left(-k_{12}+\mathrm{i} k_{3}\right) x_{3}\right)\right]\right]\right\}\right\}-\mathrm{ie}^{k_{12} x_{3}} \frac{k_{2} k_{0}^{2}}{k_{12} k^{2}}, \\
& M_{31}=M_{32}=0, \\
& M_{33}=\frac{k_{0}^{2}}{k^{2}}\left(\mathrm{e}^{\mathrm{i} k_{3} x_{3}}-\mathrm{e}^{k_{12} x_{3}}\right),
\end{aligned}
$$

where 'sign' denotes the sign function and $E_{1}$ denotes the exponential integral function of order one. The $Q_{j}$ vector is given by

$$
\begin{aligned}
& Q_{1}=Q_{2}=0 \\
& Q_{3}=2 \mathrm{i} \rho \Gamma k_{1} \frac{k_{0}^{2}}{k^{2}}\left[\frac{1}{k^{2}} \mathrm{e}^{\mathrm{i} k_{3} x_{3}}+\left(\frac{x_{3}}{2 k_{12}}-\frac{1}{2 k_{12}^{2}}-\frac{\mathrm{i} k_{3}}{k_{12} k^{2}}\right) \mathrm{e}^{k_{12} x_{3}}\right] .
\end{aligned}
$$

The corresponding expressions for turbulence in the air can be obtained by simply reflecting these expressions about the plane $x_{3}=0$ : replacing $k_{3}$ with $-k_{3}, k_{03}$ with $-k_{03}$ and $x_{3}$ with $-x_{3}$.

The $S_{j}$ vector is defined as

$$
\begin{aligned}
& S_{1}=S_{2}=0 \\
& S_{3}=-\mathrm{i} \frac{\rho_{a}}{\rho_{w}} \frac{\Gamma}{\sigma_{0}} \frac{k_{1}}{k_{12}} k_{0}^{2} \int_{0}^{t} \frac{1}{\left(k_{12}-\mathrm{i} k_{3}\right)^{2}} \sin \left(\sigma_{0}(t-s)\right) \mathrm{d} s
\end{aligned}
$$


for turbulence in the air and

$$
\begin{aligned}
& S_{1}=S_{2}=0, \\
& S_{3}=-\mathrm{i} \frac{\Gamma}{\sigma_{1}} \frac{k_{1}}{k_{12}} k_{0}^{2} \int_{0}^{t} \frac{\mathrm{e}^{\mathrm{i} \frac{\Gamma k_{1}}{2 k_{12}}(t-s)}}{\left(k_{12}+\mathrm{i} k_{3}\right)^{2}} \sin \left(\sigma_{1}(t-s)\right) \mathrm{d} s
\end{aligned}
$$

for turbulence in the water.

\section{Appendix B. Growing and oscillating parts of $\Psi$}

In (42) and (43), the terms between brackets can be expanded into a growing and an oscillating part. Expressions for these parts are presented next for turbulence in the water. The expressions for turbulence in the air may then be found by a slight modification.

After expressing the sine function in (43) in complex form, moving the factors explicitly dependent on time outside the integrals and rearranging, the term between brackets in (43) can be written

$$
\begin{aligned}
& \left|\int_{0}^{t} \frac{\mathrm{e}^{\mathrm{i} \frac{\Gamma k_{1}}{2 k_{12}} s} \sin \left(\sigma_{1}(t-s)\right)}{\left(k_{12}-\mathrm{i} k_{3}(s)\right)^{2}} \mathrm{~d} s\right|^{2} \\
& =\frac{1}{4}\left|\int_{0}^{t} \frac{\mathrm{e}^{\mathrm{i}\left(\frac{\Gamma k_{1}}{2 k_{12}}-\sigma_{1}\right) s}}{\left(k_{12}-\mathrm{i} k_{3}(s)\right)^{2}} \mathrm{~d} s\right|^{2}+\frac{1}{4}\left|\int_{0}^{t} \frac{\mathrm{e}^{\mathrm{i}\left(\frac{\Gamma k_{1}}{2 k_{12}}+\sigma_{1}\right) s}}{\left(k_{12}-\mathrm{i} k_{3}(s)\right)^{2}} \mathrm{~d} s\right|^{2} \\
& -\operatorname{Re}\left\{\frac{1}{4} \mathrm{e}^{2 \mathrm{i} \sigma_{1} t} \int_{0}^{t} \frac{\mathrm{e}^{\mathrm{i}\left(\frac{\Gamma k_{1}}{2 k_{12}}-\sigma_{1}\right) s}}{\left(k_{12}-\mathrm{i} k_{3}(s)\right)^{2}} \mathrm{~d} s \int_{0}^{t} \frac{\mathrm{e}^{\mathrm{i}\left(\frac{\Gamma k_{1}}{2 k_{12}}+\sigma_{1}\right) s}}{\left(k_{12}+\mathrm{i} k_{3}(s)\right)^{2}} \mathrm{~d} s\right\} .
\end{aligned}
$$

The third term on the right oscillates in time and was neglected in the numerical calculations, for reasons explained in Section 2.5. By making $\sigma_{1}=\sigma_{0}$ and $\Gamma=0$ in (B.1) only in the numerator of the fractions (i.e., not in $k_{3}$ ) and changing the sign of $k_{3}$, the expression applicable to the case of turbulence in the air, (42), is found: 


$$
\begin{aligned}
& \left|\int_{0}^{t} \frac{\sin \left(\sigma_{0}(t-s)\right)}{\left(k_{12}+\mathrm{i} k_{3}(s)\right)^{2}} \mathrm{~d} s\right|^{2} \\
& =\frac{1}{4}\left|\int_{0}^{t} \frac{\mathrm{e}^{-\mathrm{i} \sigma_{0} s}}{\left(k_{12}+\mathrm{i} k_{3}(s)\right)^{2}} \mathrm{~d} s\right|^{2}+\frac{1}{4}\left|\int_{0}^{t} \frac{\mathrm{e}^{\mathrm{i} \sigma_{0} s}}{\left(k_{12}+\mathrm{i} k_{3}(s)\right)^{2}} \mathrm{~d} s\right|^{2} \\
& -\operatorname{Re}\left\{\frac{1}{4} \mathrm{e}^{2 \mathrm{i} \sigma_{0} t} \int_{0}^{t} \frac{\mathrm{e}^{-\mathrm{i} \sigma_{0} s}}{\left(k_{12}+\mathrm{i} k_{3}(s)\right)^{2}} \mathrm{~d} s \int_{0}^{t} \frac{\mathrm{e}^{\mathrm{i} \sigma_{0} s}}{\left(k_{12}-\mathrm{i} k_{3}(s)\right)^{2}} \mathrm{~d} s\right\} .
\end{aligned}
$$

\section{Appendix C. Solution in terms of exponential integrals}

The product of complex conjugate time integrals in (42) and (43) was seen in appendix B to be approximately equal to the first two terms on the right of (B.1) and (B.2), due to the fact that the third terms are oscillatory. Thus, the approximate solution for the wave growth only requires a calculation of the time integrals in the non-oscillatory terms. For turbulence in the water, these are

$$
\begin{aligned}
& \int_{0}^{t} \frac{\mathrm{e}^{\mathrm{i}\left(\frac{\Gamma k_{1}}{2 k_{1} 2} \pm \sigma_{1}\right) s}}{\left(k_{12}-\mathrm{i} k_{3}(s)\right)^{2}} \mathrm{~d} s=-\frac{1}{k_{1} \Gamma}\left(\frac{\mathrm{e}^{\mathrm{i}\left(\frac{\Gamma k_{1}}{2 k_{1} 2} \pm \sigma_{1}\right) t}}{k_{3}(t)+\mathrm{i} k_{12}}-\frac{1}{k_{03}+\mathrm{i} k_{12}}\right)
\end{aligned}
$$

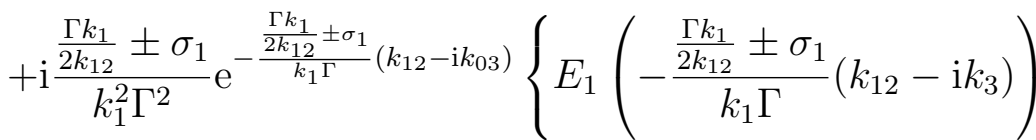

$$
\begin{aligned}
& -E_{1}\left(-\frac{\frac{\Gamma k_{1}}{2 k_{12}} \pm \sigma_{1}}{k_{1} \Gamma}\left(k_{12}-\mathrm{i} k_{03}\right)\right)+\pi \mathrm{i} H\left(\frac{\frac{\Gamma k_{1}}{2 k_{12}} \pm \sigma_{1}}{k_{1} \Gamma} k_{12}\right) \\
& \left.\times\left[\operatorname{sign}\left(\frac{\frac{\Gamma k_{1}}{2 k_{12}} \pm \sigma_{1}}{k_{1} \Gamma} k_{3}\right)-\operatorname{sign}\left(\frac{\frac{\Gamma k_{1}}{2 k_{12}} \pm \sigma_{1}}{k_{1} \Gamma} k_{03}\right)\right]\right\},
\end{aligned}
$$

and for turbulence in the air

$$
\begin{aligned}
& \int_{0}^{t} \frac{\mathrm{e}^{ \pm \mathrm{i} \sigma_{0} s}}{\left(k_{12}+\mathrm{i} k_{3}(s)\right)^{2}} \mathrm{~d} s=-\frac{1}{k_{1} \Gamma}\left(\frac{\mathrm{e}^{ \pm \mathrm{i} \sigma_{0} t}}{-k_{3}(t)+\mathrm{i} k_{12}}-\frac{1}{-k_{03}+\mathrm{i} k_{12}}\right) \\
& \pm \mathrm{i} \frac{\sigma_{0}}{k_{1}^{2} \Gamma^{2}} \mathrm{e}^{\mp \frac{\sigma_{0}}{k_{1} \Gamma}\left(k_{12}+\mathrm{i} k_{03}\right)}\left\{E_{1}\left(\mp \frac{\sigma_{0}}{k_{1} \Gamma}\left(k_{12}+\mathrm{i} k_{3}\right)\right)-E_{1}\left(\mp \frac{\sigma_{0}}{k_{1} \Gamma}\left(k_{12}+\mathrm{i} k_{03}\right)\right)\right. \\
& \left.-\pi \mathrm{i} H\left(\frac{ \pm \sigma_{0}}{k_{1} \Gamma} k_{12}\right)\left[\operatorname{sign}\left( \pm \frac{\sigma_{0}}{k_{1} \Gamma} k_{3}\right)-\operatorname{sign}\left( \pm \frac{\sigma_{0}}{k_{1} \Gamma} k_{03}\right)\right]\right\}
\end{aligned}
$$


where $H$ is the Heaviside function.

\section{References}

Batchelor, G. K., Proudman, I., 1954. The effect of rapid distortion of a fluid in turbulent motion. Quart. J. Mech. Appl. Math. 7, 83-103.

Belcher, S. E., Hunt, J. C. R., 1993. Turbulent shear flow over slowly moving waves. J. Fluid Mech. 251, 109-148.

Belcher, S. E. and Hunt, J. C. R., 1998. Turbulent flow over hills and waves. Ann. Rev. Fluid Mech. 30, 507-538.

Brocchini, M., Peregrine, D. H., 2001. The dynamics of turbulence at free surfaces. Part 1. Description. J. Fluid Mech. 449, 225-254.

Bull, M. K., 1996. Wall-pressure fluctuations beneath turbulent boundary layers: some reflections on forty years of research. J. Sound and Vib. 190, 299315.

Caulliez, G., Ricci, N., Dupont, R., 1998. The generation of the first visible wind waves. Phys. Fluids 10, 757-759.

Chang, P. A., Piomelli, U., Blake, W. K., 1999. Relationship between wall pressure and velocity-field sources. Phys. Fluids 11, 3434-3448.

Donelan, M. A., 1990. Air-sea interaction. In The sea: ocean engineering science. Volume 9. John Wiley \& Sons.

Durbin, P. A., 1978. Rapid distortion theory of turbulent flows. PhD thesis, University of Cambridge.

Gelci, R., Ramamonjiarisoa, A., Hervouet, J. Y., 1985. Génération de vagues de gravité par des allées de tourbillons aériens mobiles. J. Mécanique Théorique et Appliqué 4, 463-483.

Gemmrich, J. R., Farmer, D. M., 2004. Near-surface turbulence in the presence of breaking waves. J. Phys. Oceanogr. 34, 1067-1086.

Giovanangeli, J. P., Memponteil, A., 1985. Resonant and non-resonant waves excited by periodic vortices in airflow over water. J. Fluid Mech. 159, 69-84. 
Hasselmann, K., 1968. Weak interaction theory of ocean waves. In Basic Developments in Fluid Dynamics (ed. H. Holt), p. 117. Academic Press.

Hunt, J. C. R., 1973. A theory of flow round two-dimensional bluff bodies. J. Fluid Mech. 61, 625-706.

Hunt, J. C. R., Graham, J. M. R., 1978. Free-stream turbulence near plane boundaries. J. Fluid Mech. 84, 209-235.

Kahma, K. K., Donelan, M. A., 1988. A laboratory study of the minimum wind speed for wind wave generation. J. Fluid Mech. 192, 339-364.

Kitaigorodskii, S. A., Lumley, J. L., 1983. Wave-turbulence interactions in the upper ocean. Part I: The energy balance on the interacting fields of surface wind waves and wind-induced three-dimensional turbulence. J. Phys. Oceanogr. 13, 1977-1987.

Kondo, J., 1976. Parametrization of turbulent transport in the top meter of the ocean. J. Phys. Oceanogr. 6, 712-720.

Lee, M. J., Hunt, J. C. R., 1989. The structure of sheared turbulence near a plane boundary. 7th Simp. on Turbulent Shear Flows, Stanford.

Lee, M. J., Kim, J., Moin, P., 1990. Structure of turbulence at high shear rate. J. Fluid Mech. 216, 561-583.

Magnaudet, J., 2003. High-Reynolds number turbulence in a shear-free boundary layer: revisiting the Hunt-Graham theory. J. Fluid Mech. 484, $167-196$.

Mann, J., 1994. The spatial structure of neutral atmospheric surface-layer turbulence. J. Fluid Mech. 273, 141-168.

Miles, J. W., 1957. On the generation of surface waves by shear flow. J. Fluid Mech. 3, 185-204.

Pan, Y., Banerjee, S., 1995. A numerical study of free-surface turbulence in channel flow. Phys. Fluids 7, 1649-1664.

Phillips, O. M., 1957. On the generation of waves by turbulent wind. J. Fluid Mech. 2, 417-445.

Phillips, O.M., 1985. Spectral and statistical properties of the equilibrium 
range in wind-generated gravity waves. J. Fluid Mech. 156, 505-531.

Sazontov, A. G., Shagalov, S. V., 1984. A contribution to the theory of generation of hydrodynamic wave turbulence at an interface. Izv. Atmos. Oceanic Phys. 20, 511-517.

Teixeira, M. A. C., Belcher, S. E., 2000. Dissipation of shear-free turbulence near boundaries. J. Fluid Mech. 442, 167-191.

Teixeira, M. A. C., Belcher, S. E., 2002. On the distortion of turbulence by a progressive surface wave. J. Fluid Mech. 458, 229-267.

Thorpe, S. A., 2004. Langmuir circulation. Ann. Rev. Fluid Mech. 36, 55-79.

Townsend, A. A., 1970. Entrainment and the structure of turbulent flow. J. Fluid Mech. 41, 13-46.

Townsend, A. A., 1976. The structure of turbulent shear flow, 2nd edition. Cambridge University Press. 


\section{Figure Captions}

Fig. 1. Schematic diagram showing the flow considered for (a) turbulence in the air; (b) turbulence in the water.

Fig. 2. Time evolution of mean square slope of the waves for turbulence in the water. (a) $l=5 \mathrm{~cm}, u=5 \mathrm{~cm} \mathrm{~s}^{-1}$. Solid line: $\Gamma^{\prime}=5$, dotted line: $\Gamma^{\prime}=10$, dashed line: $\Gamma^{\prime}=20$, long-dashed line: $\Gamma^{\prime}=50$. (b) $l=5 \mathrm{~cm}, \Gamma^{\prime}=10$. Solid line: $u=3 \mathrm{~cm} \mathrm{~s}^{-1}$, dotted line: $u=5 \mathrm{~cm} \mathrm{~s}^{-1}$, dashed line: $u=7 \mathrm{~cm} \mathrm{~s}^{-1}$, longdashed line: $u=9 \mathrm{~cm} \mathrm{~s}^{-1}$. (c) $u=5 \mathrm{~cm} \mathrm{~s}^{-1}, \Gamma^{\prime}=10$. Solid line: $l=1 \mathrm{~cm}$, dotted line: $l=2 \mathrm{~cm}$, dashed line: $l=5 \mathrm{~cm}$, long-dashed line: $l=10 \mathrm{~cm}$.

Fig. 3. Time evolution of mean square slope of the waves for turbulence in the air. (a) $l=0.5 \mathrm{~m}, u=0.5 \mathrm{~m} \mathrm{~s}^{-1}$. Solid line: $\Gamma^{\prime}=50$, dotted line: $\Gamma^{\prime}=100$, dashed line: $\Gamma^{\prime}=200$, long-dashed line: $\Gamma^{\prime}=500$. (b) $l=0.5 \mathrm{~m}, \Gamma^{\prime}=100$. Solid line: $u=0.3 \mathrm{~m} \mathrm{~s}^{-1}$, dotted line: $u=0.5 \mathrm{~m} \mathrm{~s}^{-1}$, dashed line: $u=0.7 \mathrm{~m} \mathrm{~s}^{-1}$, longdashed line: $u=0.9 \mathrm{~m} \mathrm{~s}^{-1}$. (c) $u=0.5 \mathrm{~m} \mathrm{~s}^{-1}, \Gamma^{\prime}=100$. Solid line: $l=0.1 \mathrm{~m}$, dotted line: $l=0.2 \mathrm{~m}$, dashed line: $l=0.5 \mathrm{~m}$, long-dashed line: $l=1 \mathrm{~m}$.

Fig. 4. Temporal correlation of the turbulent pressure fluctuations. Solid line: $t^{\prime}=0$, dotted line: $t^{\prime}=3$, dashed line: $t^{\prime}=5$.

Fig. 5. Dimensionless wavenumber spectrum of the turbulent pressure fluctuations along the flow direction. Solid line: $t^{\prime}=0$, dotted line: $t^{\prime}=3$, dashed line: $t^{\prime}=5$.

Fig. 6. Curvature spectra of the surface waves along the flow direction, for turbulence in the water, at dimensionless time $t^{\prime}=5$. (a) $l=5 \mathrm{~cm}, u=$ $5 \mathrm{~cm} \mathrm{~s}^{-1}$. Solid line: $\Gamma^{\prime}=5$, dotted line: $\Gamma^{\prime}=10$, dashed line: $\Gamma^{\prime}=20$, long- 
dashed line: $\Gamma^{\prime}=50$. (b) $l=5 \mathrm{~cm}, \Gamma^{\prime}=10$. Solid line: $u=3 \mathrm{~cm} \mathrm{~s}^{-1}$, dotted line: $u=5 \mathrm{~cm} \mathrm{~s}^{-1}$, dashed line: $u=7 \mathrm{~cm} \mathrm{~s}^{-1}$, long-dashed line: $u=9 \mathrm{~cm} \mathrm{~s}^{-1}$. (c) $u=5 \mathrm{~cm} \mathrm{~s}^{-1}, \Gamma^{\prime}=10$. Solid line: $l=1 \mathrm{~cm}$, dotted line: $l=2 \mathrm{~cm}$, dashed line: $l=5 \mathrm{~cm}$, long-dashed line: $l=10 \mathrm{~cm}$.

Fig. 7. Variation of the curvature spectrum with direction, for turbulence in the water, for $l=5 \mathrm{~cm}, u=5 \mathrm{~cm} \mathrm{~s}^{-1}$ and $t^{\prime}=5$. Solid line: $k_{12}=20 \mathrm{~m}^{-1}$, dotted line: $k_{12}=50 \mathrm{~m}^{-1}$, dashed line: $k_{12}=100 \mathrm{~m}^{-1}$, long-dashed line: $k_{12}=200 \mathrm{~m}^{-1}$, dash-dotted line: $k_{12}=500 \mathrm{~m}^{-1}$. (a) lower shear rate, $\Gamma^{\prime}=10$. (b) higher shear rate, $\Gamma^{\prime}=50$. 


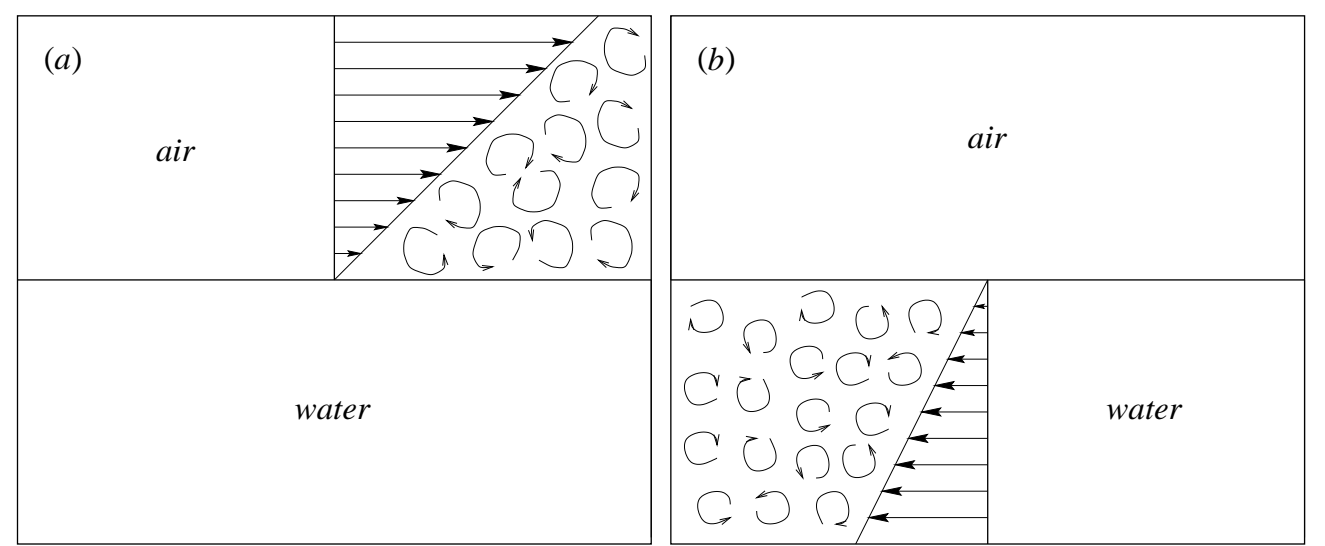

Fig. 1. Schematic diagram showing the flow considered for (a) turbulence in the air; (b) turbulence in the water. 

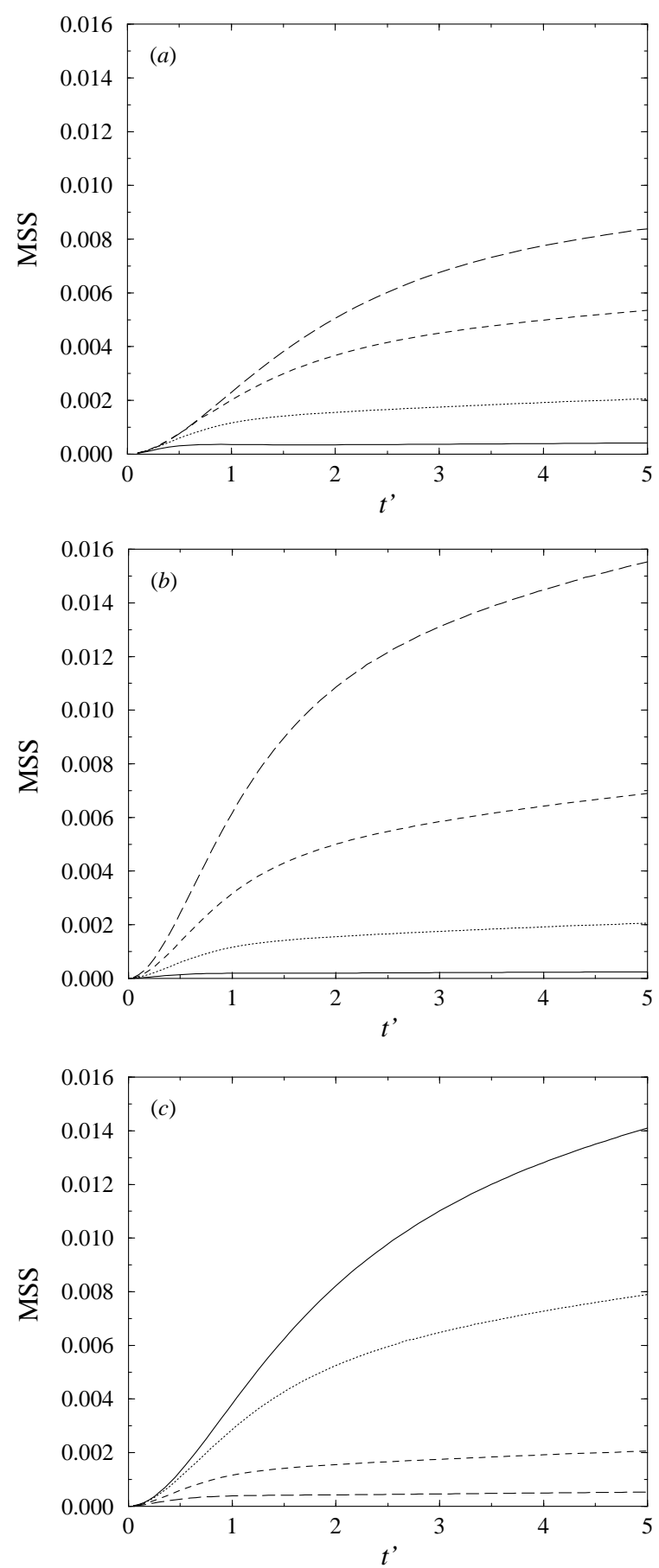

Fig. 2. Time evolution of mean square slope of the waves for turbulence in the water. (a) $l=5 \mathrm{~cm}, u=5 \mathrm{~cm} \mathrm{~s}^{-1}$. Solid line: $\Gamma^{\prime}=5$, dotted line: $\Gamma^{\prime}=10$, dashed line: $\Gamma^{\prime}=20$, long-dashed line: $\Gamma^{\prime}=50$. (b) $l=5 \mathrm{~cm}, \Gamma^{\prime}=10$. Solid line: $u=3 \mathrm{~cm} \mathrm{~s}^{-1}$, dotted line: $u=5 \mathrm{~cm} \mathrm{~s}^{-1}$, dashed line: $u=7 \mathrm{~cm} \mathrm{~s}^{-1}$, long-dashed line: $u=9 \mathrm{~cm} \mathrm{~s}^{-1}$. (c) $u=5 \mathrm{~cm} \mathrm{~s}^{-1}, \Gamma^{\prime}=10$. Solid line: $l=1 \mathrm{~cm}$, dotted line: $l=2 \mathrm{~cm}$, dashed line: $l=5 \mathrm{~cm}$, long-dashed line: $l=10 \mathrm{~cm}$. 

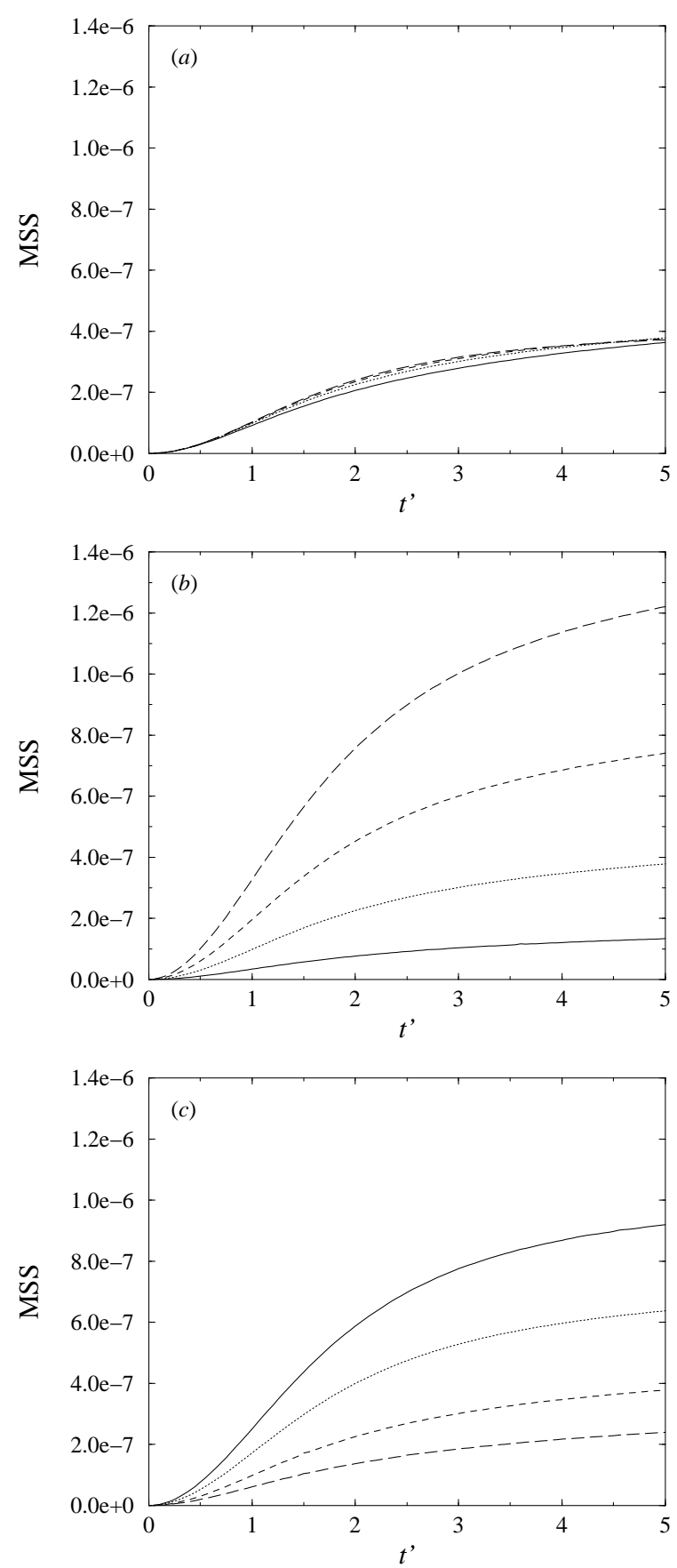

Fig. 3. Time evolution of mean square slope of the waves for turbulence in the air. (a) $l=0.5 \mathrm{~m}, u=0.5 \mathrm{~m} \mathrm{~s}^{-1}$. Solid line: $\Gamma^{\prime}=50$, dotted line: $\Gamma^{\prime}=100$, dashed line: $\Gamma^{\prime}=200$, long-dashed line: $\Gamma^{\prime}=500$. (b) $l=0.5 \mathrm{~m}, \Gamma^{\prime}=100$. Solid line: $u=0.3 \mathrm{~m} \mathrm{~s}^{-1}$, dotted line: $u=0.5 \mathrm{~m} \mathrm{~s}^{-1}$, dashed line: $u=0.7 \mathrm{~m} \mathrm{~s}^{-1}$, long-dashed line: $u=0.9 \mathrm{~m} \mathrm{~s}^{-1}$. (c) $u=0.5 \mathrm{~m} \mathrm{~s}^{-1}, \Gamma^{\prime}=100$. Solid line: $l=0.1 \mathrm{~m}$, dotted line: $l=0.2 \mathrm{~m}$, dashed line: $l=0.5 \mathrm{~m}$, long-dashed line: $l=1 \mathrm{~m}$. 


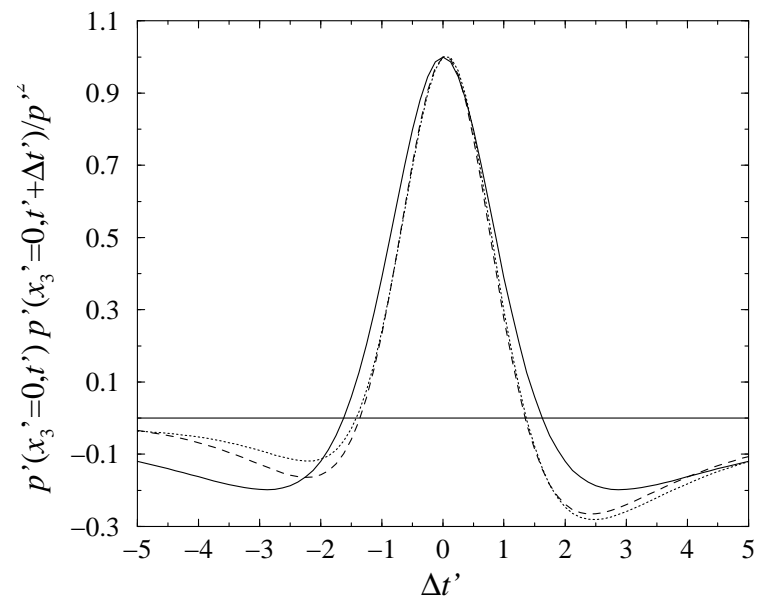

Fig. 4. Temporal correlation of the turbulent pressure fluctuations. Solid line: $t^{\prime}=0$, dotted line: $t^{\prime}=3$, dashed line: $t^{\prime}=5$. 


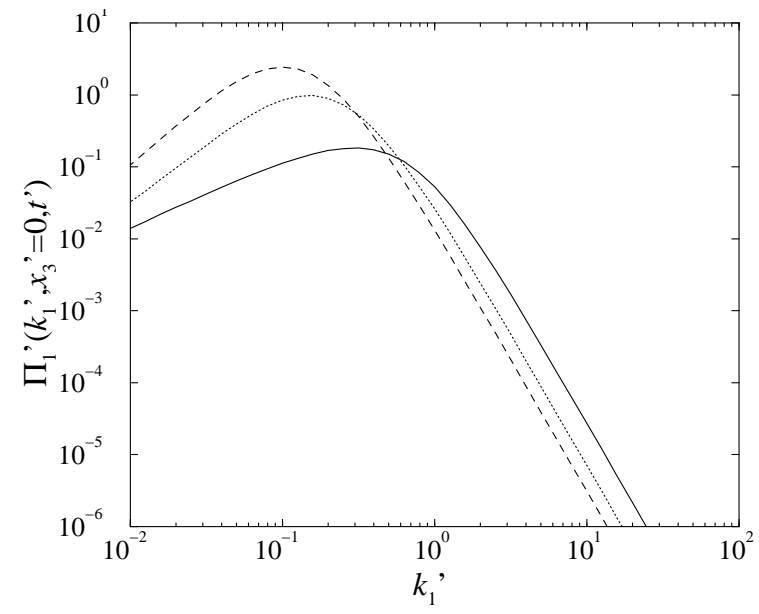

Fig. 5. Dimensionless wavenumber spectrum of the turbulent pressure fluctuations along the flow direction. Solid line: $t^{\prime}=0$, dotted line: $t^{\prime}=3$, dashed line: $t^{\prime}=5$. 

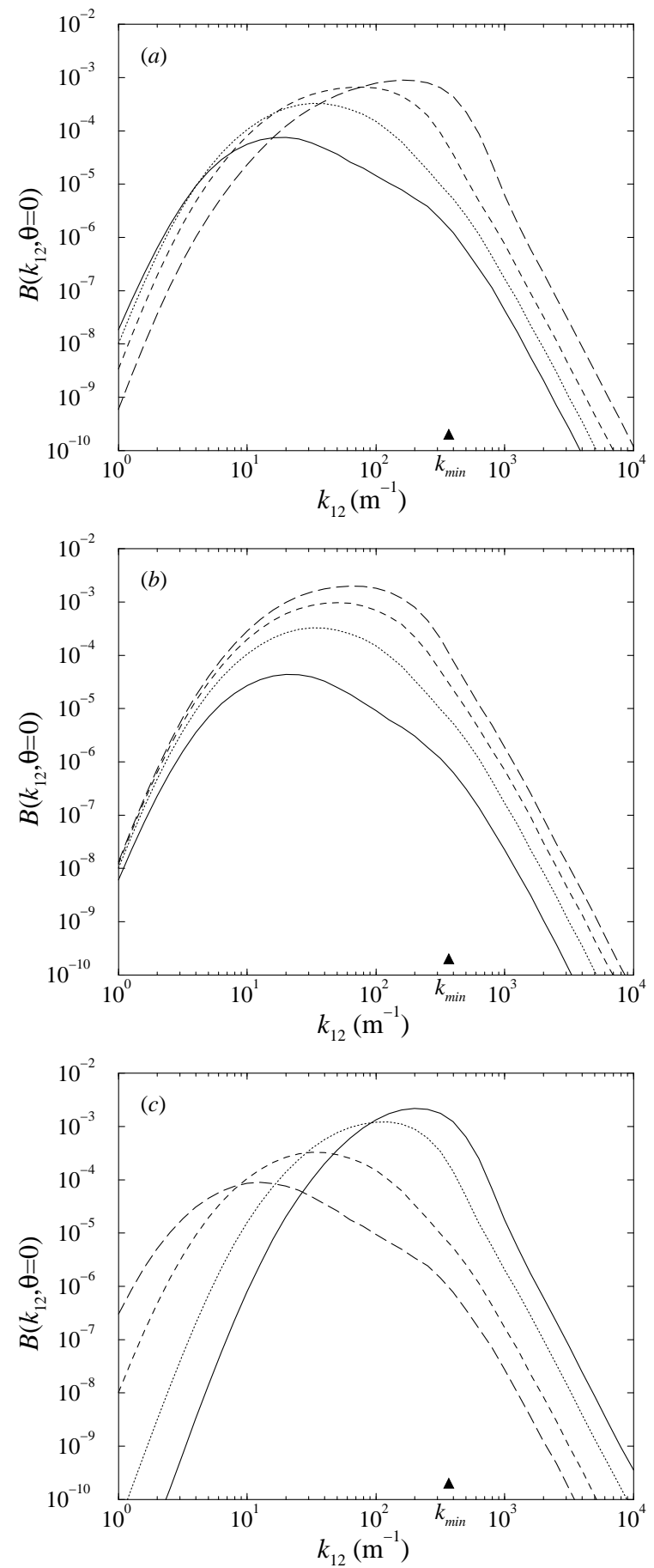

Fig. 6. Curvature spectra of the surface waves along the flow direction, for turbulence in the water, at dimensionless time $t^{\prime}=5$. (a) $l=5 \mathrm{~cm}, u=5 \mathrm{~cm} \mathrm{~s}^{-1}$. Solid line: $\Gamma^{\prime}=5$, dotted line: $\Gamma^{\prime}=10$, dashed line: $\Gamma^{\prime}=20$, long-dashed line: $\Gamma^{\prime}=50$. (b) $l=5 \mathrm{~cm}, \Gamma^{\prime}=10$. Solid line: $u=3 \mathrm{~cm} \mathrm{~s}^{-1}$, dotted line: $u=5 \mathrm{~cm} \mathrm{~s}^{-1}$, dashed line: $u=7 \mathrm{~cm} \mathrm{~s}^{-1}$, long-dashed line: $u=9 \mathrm{~cm} \mathrm{~s}^{-1}$. (c) $u=5 \mathrm{~cm} \mathrm{~s}^{-1}, \Gamma^{\prime}=10$. Solid line: $l=1 \mathrm{~cm}$, dotted line: $l=2 \mathrm{~cm}$, dashed line: $l=5 \mathrm{~cm}$, long-dashed line: $l=10 \mathrm{~cm}$. 

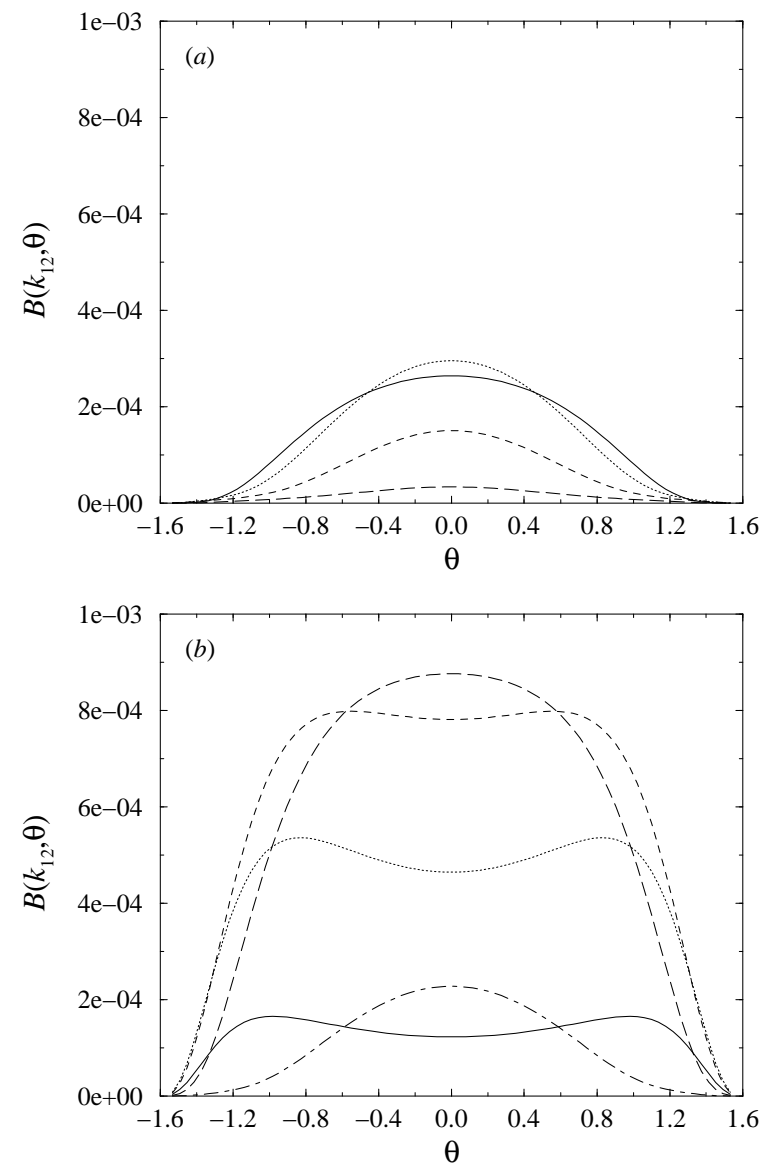

Fig. 7. Variation of the curvature spectrum with direction, for turbulence in the water, for $l=5 \mathrm{~cm}, u=5 \mathrm{~cm} \mathrm{~s}^{-1}$ and $t^{\prime}=5$. Solid line: $k_{12}=20 \mathrm{~m}^{-1}$, dotted line: $k_{12}=50 \mathrm{~m}^{-1}$, dashed line: $k_{12}=100 \mathrm{~m}^{-1}$, long-dashed line: $k_{12}=200 \mathrm{~m}^{-1}$, dash-dotted line: $k_{12}=500 \mathrm{~m}^{-1}$. (a) lower shear rate, $\Gamma^{\prime}=10$. (b) higher shear rate, $\Gamma^{\prime}=50$. 